4 Ganfei $\mathrm{Xu}^{1,2, \dagger}$, Weiyi Huang ${ }^{1, \dagger}$, Shaoqian $\mathrm{Du}^{1, \dagger}$, Minjing Huang ${ }^{2, \dagger}$, Jiacheng $5 \quad$ Lyu $^{2}$, Fei Zhou ${ }^{1}$, Rongxuan Zhu ${ }^{1}$, Yuan Cao ${ }^{1}$, Jingxuan $\mathrm{Xv}^{1}$, Ning $\mathrm{Li}^{1}$, Guoying $6 \mathrm{Yu}^{3}$, Binghua Jiang ${ }^{4}$, Olivier Gires ${ }^{5}$, Lei Zhou ${ }^{6}$, Hongwei Zhang ${ }^{7, \star}$, Chen 7 Ding ${ }^{2,8, \star *}$, Hongxia Wang ${ }^{1, \star \star *}$

\section{Proteomic Characterization of Serum Small Extracellular Vesicles in Human Breast Cancer}

9 State Key Laboratory of Oncogenes and Related Genes, Department of Oncology, Shanghai General Hospital, Shanghai Jiao Tong University School of Medicine, Shanghai, China

2 State Key Laboratory of Genetic Engineering and Collaborative Innovation Center for 3 Genetics and Development, School of Life Sciences, Institute of Biomedical Sciences, 4 Human Phenome Institute, Fudan University, Shanghai 200433, China

53 State Key Laboratory of Cell Differentiation and Regulation, Henan International Joint 6 Laboratory of Pulmonary Fibrosis, Henan Center for Outstanding Overseas Scientists 17 of Pulmonary Fibrosis, College of Life Science, Institute of Biomedical Science, $8 \quad$ Henan Normal University, Xinxiang 453007, China

94 Academy of Medical Science, Zhengzhou University, Zhengzhou 450001, China.

5 Institute of Molecular Medicine, Renji Hospital, Shanghai Jiao Tong University School 1 of Medicine, Shanghai 200127, China

6 Singapore Eye Research Institute, The Academia, 20 College Road, Discovery Tower Level 6, Singapore 169856, Singapore

4 Department of General Surgery, Zhongshan Hospital, Fudan University. 180 Fenglin Road, Shanghai 200032

8 Department of Anatomy and Neuroscience Research Institute, School of Basic 7 Medical Sciences, Zhengzhou University, Zhengzhou 450001, China

8 † These authors contributed equally to this work 
bioRxiv preprint doi: https://doi org/10.1101/2021.1126.470104; this version posted November 27, 2021. The copyright holder for this

preprint (which was not certified by peer review) is the author/funder, who has granted bioRxiv a license to display the preprint in perpetuity. It is made available under aCC-BY-NC-ND 4.0 International license.

29 * Corresponding authors. Tel: 021-63240090; Email: zhang.hongwei@zs-hospital.sh.cn

$30 \quad{ }^{* *}$ Corresponding authors. Tel: 021-31246742; Email: chend@fudan.edu.cn

$31 \quad{ }^{* * *}$ Corresponding authors. Tel: 021-63240090; Email: whx365@126.com

32

33 Key Words: Breast cancer; Small extracellular vesicles; Lymph node

34 metastases; Distant metastasis; Proteomics

35 
Abstract

37 There is a lack of comprehensive understanding of breast cancer (BC) specific

38 sEVs characteristics and composition on BC unique proteomic information

39 from human samples. Here, we interrogated the proteomic landscape of sEVs

40 in 167 serum samples from patients with BC, benign mammary disease (BD)

41 and from healthy donors (HD). The analysis provides a comprehensive

42 landscape of serum sEVs with totally 9,589 proteins identified, considerably

43 expanding the panel of sEVs markers. Of note, serum BC-sEVs protein

44 signatures were distinct from those of $\mathrm{BD}$ and HD, representing stage- and

45 molecular subtype-specific patterns. We constructed specific sEVs protein

46 identifiers that could serve as a liquid biopsy tool for diagnosis and

47 classification of BC from benign mammary disease, molecular subtypes, as

48 well as assessment of lymph node metastasis. We also identified 11 potential

49 survival biomarkers for distant metastasis. This work may provide reference

50 value for the accurate diagnosis and monitoring of $\mathrm{BC}$ progression using

51 serum sEVs. 


\section{Introduction}

54 Breast cancer $(\mathrm{BC})$ is one of the most common cancers worldwide and 55 accounts for $30 \%$ of female cancers (Kim et al, 2012; Liu et al, 2021; Siegel et 56 al, 2021). A long-term decline in the death rate has been observed since the 57 mid-1970s due to improvements in treatment protocols, including the development of chemotherapy, immunotherapy and targeted therapies. However, improvements in clinical outcomes have slowed over the past

60 decade, and distant metastasis remains the major cause of mortality (Cassetta

61 \& Pollard, 2017; Liu et al, 2019a; Siegel et al., 2021; Yin et al, 2014; Zhu et al, 62 2019). The early detection and dynamic assessment of the metastatic status of $63 \mathrm{BC}$ patients are of great value for the treatment and longitudinal analysis of 64 cancer evolution in response to therapy. To achieve this, liquid biopsies utilizing molecular classifiers detected in blood from patients, such as circulating tumor cells, circulating free DNA, and exosomes, offer minimal invasiveness, fewer complications, and an increased ability for longitudinal monitoring compared with traditional tumor tissue biopsies (Wan et al, 2017; Yoneda et al, 2019). More importantly, liquid biopsy is more informative than

70 single locally restricted biopsies, providing unique information about tumor 71 heterogeneity, clonal evolution, and the potential development of 72 premetastatic cancer cells (Hoshino et al, 2020).

73 Circulating small extracellular vesicles (sEVs), such as exosomes or 74 exosome-like vesicles (ELVs), are 30-150 $\mathrm{nm}$ in size and carry a restricted set 75 of nucleic acids, lipids, and proteins (Balaj et al, 2011; Johnstone et al, 1987; 76 Kim et al, 2013; Peinado et al, 2011; Raposo \& Stoorvogel, 2013; Skog et al, 77 2008; Thakur et al, 2014; Thery et al, 2009; Valadi et al, 2007; Wang \& Gires, 78 2019) that contribute to intercellular communication in normal physiology and 79 pathology (Johnstone et al., 1987; Maas et al, 2017; Skog et al., 2008; 80 Yanez-Mo et al, 2015). The functional importance of sEVs has been 
81 intensively studied in multiple human cancers, including BC (Hoshino et al.,

82 2020). Increasing evidence suggests that sEVs are actively released from

83 cancer cells and markedly affect the tumor microenvironment (TME) as well as

84 the immune ecosystem (Huber et al, 2005), thereby constructing distant

85 metastatic niches and facilitating cancer growth (Fang et al, 2018; Kralj-Iglic,

86 2012; Ozer et al, 2020) and metastasis (Chen et al, 2018; Costa-Silva et al,

87 2015; Hoshino et al, 2015; Peinado et al, 2012; Zhang \& Wang, 2015). Of note,

88 the membrane encapsulation of sEVs promotes their structural integrity, and

89 cargos located within sEVs are more stable than other serological proteins

90 since they have protection against degradation by circulating proteases and

91 other enzymes (Li et al, 2017a). Considering their facilitated retrieval and their

92 relatively ubiquitous presence and abundance in serum, sEVs can provide

93 ample materials for downstream analysis in BC detection, prognosis, and

94 therapeutic monitoring as a promising, noninvasive liquid biopsy approach

95 (Choi et al, 2021; Lee et al, 2018; Li et al, 2017b; Wang et al, 2018). For

96 instance, Peinado et al. showed that an "sEV protein signature" could identify

97 melanoma patients at risk for metastasis to nonspecific distant sites (Peinado

98 et al., 2012). Hoshino et al. identified a specific repertoire of integrins

99 expressed on cancer-derived sEVs, which were distinct from cancer cells, that

100 dictated exosome adhesion to specific cell types and ECM molecules in

101 particular organs (Hoshino et al., 2015).

102 The sEV proteome has been proposed to offer unique advantages as an

103 informative readout for the detection and stratification of BC (Rontogianni et al,

104 2019). Nonetheless, the challenge is to optimize a proteomic profiling

105 approach for sEVs to define and standardize reliable methods. Despite the

106 availability of several public sEV protein databases (e.g., Vesiclepedia

107 (www.microvesicles.org/) (Kalra et al, 2012), EVpedia (www.evpedia.info)

108 (Kim et al., 2012) and ExoCarta (www.exocarta.org) (Kim et al., 2013)), much 
109 remains unknown about the sEV proteomes of BC. This includes the definition

110 of (1) markers to distinguish BC from benign disease and healthy state, (2)

111 markers to distinguish diverse molecular subtypes of invasive breast cancer

112 (IBC), (3) markers to predict lymph node (LN) metastases, and (4) the open

113 question of whether molecules present on IBC-derived sEVs are "addressing"

114 them to specific organs. These unresolved problems highlight the need for a

115 better understanding of the protein composition of BC-derived sEVs that could

116 qualify them as biomarkers for clinical application, with a specificity and

117 sensitivity mostly superior to those of traditional serum markers. To address

118 these aims, mass spectrometry-based proteomic profiling is emerging as a

119 strategy to gain insight into the biological cargos, functions, and clinical

120 potential of sEVs (Wang et al, 2020).

121 Here, we applied a mass spectrometry-based, data-independent acquisition

122 (DIA) quantitative approach to determine the proteomic features of human

123 serum sEVs derived from patients with BC, benign mammary disease (BD),

124 and healthy donors (HDs). In total, we identified 9,589 proteins from 167

125 analyzed samples with a mean of 1,695 proteins quantified per sEV sample.

126 Classification of the pathways related to the enriched proteins revealed that

127 proteins preferentially packaged in BC-sEVs correlated with interferon

$128 \quad \mathrm{Y}$-mediated signaling as well as pathways associated with immune response

129 regulation, antigen processing and presentation, glycolysis and angiogenesis.

130 By examining the SEV proteomes, we constructed specific sEV protein

131 identifiers that could serve as a liquid biopsy tool for the diagnosis and

132 classification of $\mathrm{BC}$ from $\mathrm{BD}$ and its molecular subtypes, as well as the

133 assessment of LN metastasis. Of note, we found that adipocytes play an

134 important role in the LN metastasis of BC. We also identified 11 potential

135 survival markers for distant BC metastasis and 2 potential survival markers for

136 lung metastasis. This work may provide reference value for the accurate 
137 diagnosis and monitoring of $\mathrm{BC}$ progression using serum sEVs, and the

138 identification of novel molecules packaged in sEVs offers an opportunity for

139 the targeted therapy of $\mathrm{BC}$ in the future.

141 Results

\section{Proteomic characterization of BC-derived sEVs}

143 To elucidate the proteomic profile of BC-derived sEVs, we purified sEVs from

144167 human serum samples derived from BC patients $(n=126)$, BD patients $(n$

$145=17$ ), and HDs ( $n=24)$ by differential ultracentrifugation as described in the

146 Methods and in accordance with previously reported protocols (Colombo et al,

147 2014; Peinado et al., 2012; Xu et al, 2016) (Fig 1A and C). All samples were

148 collected prospectively from treatment-naive stage I-IV BC patients (Fig 1B,

149 Appendix Table 1). Under transmission electron microscopy (TEM) in 150 combination with nanoparticle tracking analysis (NTA), the isolated sEVs

151 appeared as morphologically uniform vesicular structures $30-150 \mathrm{~nm}$ in size

152 surrounded by a double-layer membrane (Fig 1D, Appendix Fig S1A). sEV

153 samples were verified by immunoblotting analyses using the conventional

154 markers CD9, CD63, TSG101, and ALIX, while we examined 24 sEV markers

155 in our proteomics data (Hoshino et al., 2020) (Fig 1E, Appendix Fig S1B).

156 Clinical data, including sex, age at diagnosis, tumor staging, BC subtypes, LN

157 status, distant metastasis, and survival, are summarized in Fig 1B and Table

158 S1.

159 A proteomic database of serum sEVs was constructed using label-free 160 LC-MS/MS analysis, identifying 9,589 proteins in total from the 167 analyzed 161 samples at a protein- and peptide-level FDR of less than 5\% (Fig 1F). The 162 protein abundance was first calculated by iBAQ and then normalized as FOT,

163 allowing for comparison among different experiments. The mean number of 164 proteins detected per sEV sample was 1,695 (range 793 to 2,253 proteins) 
165 (Fig 1G). In general, 1,924, 187, and 145 unique sEV proteins were identified 166 in BC, BD, and HD samples, respectively (Fig 1H). Globally, the dynamic

167 range of proteins detected spanned eight orders of magnitude (Appendix Fig

168 S1C). Collectively, these data were consistent with previous reports that sEV 169 protein profiles differ significantly depending on the sample source (Wu et al, 170 2019), and SEVs released by BC cells and from other cancer cells may carry 171 more encapsulated cargos for signal transfer to induce the malignant

172 transformation and proliferation of recipient cells (Milane et al, 2015).

BC-derived sEVs exhibited specific signatures related to immune

175

176

\section{response, metabolism, and metastasis}

Next, proteomic data were analyzed to determine the characteristics of BC-derived SEVs. PCA demonstrated a clear distinction among the three different types of samples, which further highlighted the diverse proteomic patterns among BC-, BD-, and HD-sEVs that underpinned our stratification analysis (Appendix Fig S2A).

To decipher the protein network associated with BC tumorigenesis, we identified 287,602 , and 112 proteins that were significantly overrepresented in the $\mathrm{BC}\left(\mathrm{BC}_{\text {mean }} / \mathrm{BD}_{\text {mean }}>2\right.$-fold and $\mathrm{BC}_{\text {mean }} / \mathrm{HD}_{\text {mean }}>2$-fold $), \mathrm{BD}$ $\left(\mathrm{BD}_{\text {mean }} / \mathrm{BC}_{\text {mean }}>2\right.$-fold and $\mathrm{BD}_{\text {mean }} / \mathrm{HD}_{\text {mean }}>2$-fold $)$ and $\mathrm{HD}\left(\mathrm{HD}_{\text {mean }} / \mathrm{BC}_{\text {mean }}>\right.$ 2-fold and $\mathrm{HD}_{\text {mean }} / \mathrm{BD}_{\text {mean }}>$ 2-fold) samples, respectively (see Materials and Methods). Clustering and cluster-specific enrichment analyses of these proteins using GOBP and Reactome pathway annotations showed that these differentially enriched proteins were involved in distinctive biological processes and pathways (Fig 2A, Appendix Table 2). Specifically, COPI-mediated anterograde transport (Fisher's exact test, $p=3.88 \mathrm{e}-3$ ), vesicle-mediated transport (Fisher's exact test, $p=1.26 \mathrm{e}-4$ ), and regulation of actin dynamics for phagocytic cup formation-related proteins (Fisher's exact test, $p=2.48 \mathrm{e}-5$ ) 
193 (i.e., ADD2, ARF5, ARPC1A, IGHV3-53, IGHV4-39, SSC5D, and COPE) were

194 enriched in HD samples (Fig 2A and B, Appendix Table 2). BD-sEVs were characterized by proteins related to cell-cell adhesion (Fisher's exact test, $p=$ 2.98e-19) (i.e., STAT1, PTPN1, RPL24, and FNBP1L), cholesterol metabolic process (Fisher's exact test, $p=3.34 \mathrm{e}-7$ ) (i.e., PON1, APOC1, APOA2, ANGPTL3, and LIPC), and response to estrogen (Fisher's exact test, $p=$ 2.13e-2) (i.e., F7, LDHA, HSP90AA1, IGFBP2, and CTNNA1) (Fig 2A and B,

200 Appendix Table 2). Of note, BC-sEVs exhibited specific signatures related to 201 the immune response, metabolism, and metastasis, potentially reflecting the functional roles and molecular heterogeneity of sEVs during BC tumorigenesis and progression. Classification of the pathways related to the enriched 204 proteins from BC-sEVs revealed that these selectively packaged proteins are 205 involved in the interferon $y$-mediated signaling pathway (Fisher's exact test, $p$ = 4.94e-4) (i.e., HCK, HLA-H, HLA-B, HLA-C, HLA-A, HLA-G, and CD44), 207 regulation of immune response (Fisher's exact test, $p=4.61 \mathrm{e}-5$ ) (i.e., 208 IGLV3-25, COL3A1, CXADR, IGLV3-27, HLA-A, IGLV7-43, and PVR), antigen 209 processing and presentation (Fisher's exact test, $p=1.16 \mathrm{e}-5)$ (i.e., ITGB1, 210 IGLV3-25, CXADR, IGLV3-27, IGLV7-43, PVR, and HLA-G), glycolytic 211 process (Fisher's exact test, $p=1.29 \mathrm{e}-3$ ) (i.e., GPI, PGK1, PGAM4, PGK2, 212 and PGM1), and angiogenesis (Fisher's exact test, $p=3.92 e-2$ ) (i.e., GPI, 213 RNF213, ANGPTL6, MMP2, PECAM1, CYP1B1, NAA15, and TYMP) (FC > 2, 214 one-way ANOVA $p<0.05$ ) (Fig $2 \mathrm{~A}$ and B, Appendix Table 2). Notably, in the 215 Tang et al. BC cohort (Tang et al, 2018), among sEV proteins that were 216 specifically highly expressed in BC samples, patients with high expression of 217 MMP2 and TYMP appeared to have poor prognostic outcomes (log rank test, $p$ $218<0.05)($ Fig 2C). These findings that BC-, BD-, and HD-sEV cargos are distinct 219 and related to singular cellular processes suggest that sEV protein packaging into $\mathrm{SEV}$ s is heterogeneous and reflects $\mathrm{BC}$ biology. 
222 Specific damage-associated molecular pattern (DAMP) molecules are packaged in BC-derived sEVs

224 Recent advances have indicated that DAMP molecules, such as nucleic acids, 225 histones, high mobility group box 1, S100, and heat shock proteins, act as 226 endogenous ligands of innate immune receptors and are linked to the immune 227 response and cancer progression (Becker et al, 2016). In total, we identified 228210 different DAMPs in all sEV datasets (Appendix Fig S2B, Appendix Table 229 2). Specifically, the analysis identified 197, 145, and 157 DAMPs in BC-, BD-, 230 and HD-sEVs, respectively, suggesting that more DAMPs were enriched in BC 231 samples than in BD and HD samples (Appendix Fig S2B). Thirty-two of these 232 DAMPs were identified only in BC-sEVs, 9 DAMPs only in BD-sEVs, and 4 233 DAMPs only in HD-sEVs (Appendix Fig S2B). Of all DAMPs identified in 234 BC-sEVs, 27 DAMPs (e.g., ACAN, ANXA11, and CD44) were shared by > 50\% 235 of BC samples and were enriched compared to BD-sEVs and/or HD-sEVs (Fig 236 2D).

237 Among them, 9 DAMPs, including aggrecan (ACAN), annexin A11 238 (ANXA11), CD44, fibrinogen gamma chain (FGG), integrin-linked kinase (ILK), 239 LGALS3, and several ITGs (ITGA6, ITGB1, and ITGB3), were exclusively 240 present in BC-sEVs versus BD- and HD-sEVs, suggesting that they are 241 specific sEV markers in BC development and progression (Fig 2D). ITGA6, 242 ITGB1, and ITGB3 are members of the integrin family of proteins involved in 243 cell adhesion and recognition in a variety of processes, including tissue repair, 244 hemostasis, immune response, and metastatic dissemination of cancer cells 245 (Laudato et al, 2017; Wang et al, 2019b). ANXA11 and LGALS3 are 246 associated with the progression of some cancers (Liu et al, 2019b; Wang et al, 247 2019a). Another 10 DAMP proteins were highly enriched in both BC- and 
249 S100A9, and TXNDC5, whereas they were rarely detected in HD samples,

250 suggesting that they represent sEV DAMPs shared across BC and BD (Fig 2E).

251 Interestingly, our analyses revealed that 8 DAMP molecules (A2M, ANXA5,

252 CALR, FGB, IL6ST, LGALS3BP, LYVE1, and S100A7) were abundantly

253 expressed in both BC- and HD-sEVs (Fig 2E). This finding is consistent with

254 previous studies reporting that the noncancer-derived sEV proteome is as

255 informative as the cancer-derived sEV proteome in specific cancer types

256 (Hoshino et al., 2020). It is worth noting that 6 of these molecules (ANXA11,

257 ILK, ITGA6, ITGB1, LGALS3, and OMD) were highly expressed in BC and 258 were associated with poor prognosis in the Tang et al. BC cohort (Tang et al., 259 2018) (Fig 2E, Appendix Fig S2C).

Possible intercellular communication network diagram of BC-driven sEVs in the TME

263 Previous evidence suggests that sEVs interact with recipient immune cells to 264 participate in TME remodeling, an effect that is mediated by encapsulated molecular cargos derived from parent cancer cells (Becker et al., 2016). Thus, 266 the proteomics profile of BC-sEVs may reflect the status of corresponding 267 immune cells in the TME. To further map the differentially enriched sEV 268 proteins to the immune response, we performed cell type deconvolution analysis using xCell (Aran et al, 2017). A heatmap of overall and type-specific

270 enrichment scores was constructed to identify the immune landscape of BC 271 (Fig 2F). Specifically, the enrichment scores of macrophages M2, adipocytes, 272 epithelial cells, CD4+ T cells, $\mathrm{y} \delta \mathrm{T}$ cells (Tgd), Th2 cells and 273 megakaryocyte-erythroid progenitor cells (MEPs) were significantly elevated in 274 BC-sEVs compared to HD-sEVs, with FC > 1.3 and Student's $t$ test $p$ value < 2750.05 (Fig 2F, Appendix Table 2). The analysis suggested a possible intercellular communication network of BC-driven sEVs in the TME when we 
277 inferred the relative abundance of various immune cell subtypes in the TME.

278 MEPs represent a bipotent transitional state that is permissive to the 279 generation of unipotent progenitors of megakaryocytic or erythroid lineages 280 (Xavier-Ferrucio et al, 2019). Adipocytes in the TME play dynamic and 281 sophisticated roles in facilitating BC development (Cao, 2019). These 282 BC-derived sEVs may impact the TME by promoting tumor cell growth and 283 progression, modulating immune responses, regulating angiogenesis and 284 inducing metastatic behavior through MEPs, endothelial cells, and mv 285 endothelial cells (Fig 2G).

Eight-protein diagnostic model to distinguish BC from BD and the healthy population

289 To further assess whether sEV proteins could be used as a liquid diagnostic tool to discriminate cancers from noncancers, we next sought to determine

291 shared and unique sEV proteins by performing pairwise comparisons of 292 proteomes between BC-, BD-, and HD-sEVs. We applied the XGBoost 293 classifier, which is robust to noise and overfitting, to verify a distinct sEV 294 protein subset that can accurately distinguish the BC, BD and HD samples.

295 To train and subsequently test the model, sEV samples were evenly 296 partitioned based on the sample source, and $70 \%$ of samples were used as a 297 training set, with the remaining $30 \%$ used as an independent test set. Applying 298 5-fold cross-validation to the training set, a combination of 8 sEV proteins 299 (STAT1, PON1, APOC1, APOC2, MMP2, IGHV4-39, IGHV3-53, and ADD2) 300 was used to construct a signature that yielded a sensitivity of $100 \%$ and 301 specificity of $100 \%$ for discriminating BC from BD and HD (Fig $2 \mathrm{H}-\mathrm{I}$, Appendix 302 Fig S2D). Notably, when applying this eight-protein identifier to sEV samples 303 of the independent test set, the model achieved $97 \%$ sensitivity and $83 \%$ specificity in the diagnosis of $\mathrm{BC}(\mathrm{Fig} 2 \mathrm{H})$. 
306 Proteomic characteristics of sEVs derived from four clinical subtypes of 307 BC

308 IBC is a highly heterogeneous disease that can be categorized into various 309 intrinsic or molecular subtypes, which are differentially correlated with clinical 310 presentation, prognosis, distant metastasis, and response to therapy. 311 Molecular subtypes are defined based on the gene expression signature and 312 protein expression of estrogen receptor (ER), progesterone receptor (PR), 313 human epidermal growth factor receptor 2 (Her2), and proliferative cell nuclear 314 antigen (Ki67) (Li et al, 2021; Peng et al, 2019; Vallejos et al, 2010). We 315 reasoned that since the biological behavior of IBC cells differs significantly 316 among IBC subtypes, biological cargos carried by sEVs may vary among 317 diverse molecular subtypes. To distinguish proteomic landscapes among 318 diverse molecular subtypes of IBC and identify drivers that boost intertumoral 319 heterogeneity and cancer evolution, we analyzed sEV samples from luminal A 320 (ER+/PR+, low-grade and low-Ki67 index, $n=20)$, luminal $B(E R+/ P R+$ of 321 higher grade and proliferative index, $n=50$ ), Her2-enriched (Her2+ with or 322 without $E R, n=21$ ), and triple-negative (ER-PR-Her2-, TNBC, $n=23$ ) IBCs in 323 our cohort. PCA demonstrated a clear distinction among the different 324 molecular subtypes, which further highlighted the distinct proteomic patterns among several clinical subtypes of IBC samples (Appendix Fig S3A).

326 Next, we applied a $t$ test with a nominal $p$ value cut-off of $<0.05$ and 327 identified 87, 82, 83, and 104 sEV proteins that were significantly 328 overrepresented in luminal A (FC (luminal A/any of the other three subtypes) > 329 2), luminal B (FC (luminal B/ any of the other three subtypes) > 2), 330 Her2-enriched (FC (Her2-enriched/ any of the other three subtypes) > 2), and 331 TNBC (FC (TNBC/ any of the other three subtypes) > 2) samples (see 332 Materials and Methods). Clustering and cluster-specific enrichment analyses 
333 of the enriched proteins using GOBP and KEGG pathway annotations showed

334 the distinctive biological processes and pathways represented in luminal $A$, 335 luminal B, Her2-enriched, and TNBC samples (Fig 3A and B, Appendix Table 336 3). Specifically, luminal A-derived sEVs were characterized by proteolysis 337 involved in cellular protein catabolic processes (i.e., PSMB7, PSMB2, FAP, 338 and CAPN2) (Fisher's exact test, $p=1.23 \mathrm{e}-3$ ) and positive regulation of 339 protein insertion into mitochondrial membrane involved in apoptotic signaling 340 pathway (i.e., YWHAB, YWHAG, and YWHAH) (Fisher's exact test, $p=$ 341 7.69e-3). Luminal B-derived sEVs were characterized by cellular response to 342 insulin stimulus (i.e., RAB10, PKLR, GOT1, and STAT1) (Fisher's exact test, $p$ $343=4.41 \mathrm{e}-3$ ) and response to hypoxia (i.e., ALAD, VCAM1, PKLR, and HSPD1) 344 (Fisher's exact test, $p=3.77 \mathrm{e}-2)$. Her2-enriched sEV-enriched proteins were 345 related to cellular response to reactive oxygen species (i.e., PRDX1, TXN, and 346 SOD3) (Fisher's exact test, $p=1.34 \mathrm{e}-2$ ), glucose metabolic process (i.e., 347 FABP5, GAA, BPGM, and GAPDH) (Fisher's exact test, $p=3.47 \mathrm{e}-3$ ), and 348 keratinization (i.e., CASP14, KRT17, and TGM3) (Fisher's exact test, $p=$ 349 1.99e-2). TNBC samples were characterized by platelet degranulation (i.e., 350 AHSG, ACTN4, PPBP, TLN1, and PF4) (Fisher's exact test, $p=2.67 \mathrm{e}-3$ ), 351 blood coagulation (i.e., EHD1, COL1A1, PROC, COL1A2, F11, and PRKACB) 352 (Fisher's exact test, $p=3.74 \mathrm{e}-3$ ), adaptive immune response (i.e., DBNL, 353 ANXA1, ERAP2, and ICOSLG) (Fisher's exact test, $p=5.03 e-2)$, and platelet 354 activation (i.e., COL1A1, COL1A2, SAA1, and PF4) (Fisher's exact test, $p=$ 355 2.67e-2) (Fig 3B and C, Appendix Table 3).

356 Collectively, these data suggested that proteomic profiles of serum-derived 357 sEVs reflect selective packaging, which represents an informative readout and 358 differs among diverse subtypes of BCs. 
361 To further investigate the clinical significance of the differentially enriched 362 protein cargos, we addressed whether they could be utilized as a novel liquid 363 biopsy method to distinguish diverse clinical subtypes in clinical practice. 364 Employing XGBoost classification, which is robust to noise and overfitting, we 365 constructed a 61-protein classifier model that can accurately discriminate the 366 Iuminal A, luminal B, Her2-enriched, and TNBC subtypes. To train and subsequently test the model, $70 \%$ of samples were used as a training set, with 368 the remaining $30 \%$ used as an independent test set, in the same manner as 369 previously described. Similar to our analysis of BC versus non-BC-sEVs, we constructed a 61-protein classifier model using the XGBoost classifier. To test 371 the 61-sEV protein model, 5-fold cross-validation of the training set was 372 performed and yielded a sensitivity of $100 \%$ and a specificity of $100 \%$ for each 373 molecular subtype (Fig 3D, Appendix Fig S3B). When applying the 61-protein classifier to the independent test set, the model achieved $67 \%$ sensitivity and $37597 \%$ specificity in the diagnosis of luminal A, $80 \%$ sensitivity and $70 \%$ 376 specificity in diagnosis of luminal B, $57 \%$ sensitivity and $89 \%$ specificity in 377 diagnosis of Her2-enriched, and $71 \%$ sensitivity and $100 \%$ specificity in 378 diagnosis of TNBC (Fig 3D, Appendix Fig S3B). The receiver operating 379 characteristic $(\mathrm{ROC})$ curve derived from the 61-protein signature showed good 380 sensitivity and specificity, with an area under the curve (AUC) of 1.0 (Fig 3E). 381 Then, the 61-protein signature was validated in the test set, resulting in a ROC 382 curve with an AUC of 0.875 (Fig 3E).

383 Thus, serum sEV proteomes can be beneficial in determining the BC 384 subtype for dynamic monitoring in patients during tumor progression, avoiding 385 repeated tissue biopsies. 
388 Furthermore, to elucidate the mechanism of LN metastasis in IBC, we 389 analyzed sEV proteins of IBC patients with LN metastases (IBC_LN, $\mathrm{n}=51$ ) 390 and without LN metastases (IBC_Pure, $\mathrm{n}=54$ ). PCA clearly distinguished 391 between IBC_LN and IBC_Pure samples at the protein level, which further 392 highlighted the diverse proteomic patterns between sEVs from IBC_LN and 393 IBC_Pure samples (Appendix Fig S4A). We applied Student's $t$ test with a 394 nominal $p$ value cut-off of $<0.05$ and identified significantly enriched 395 sEV-derived proteins in IBC_LN compared with IBC_Pure (FC > 2). The 396 results are summarized in the volcano plot shown in Fig S4B, and the most 397 prominent proteins are indicated (Appendix Fig S4B).

398 We further performed clustering and cluster-specific enrichment analyses of 399 the upregulated proteins using gene set enrichment analysis (GSEA). We 400 found that IBC_LN samples were characterized by proteins related to 401 hallmarks of adipogenesis (Fig 4A). To investigate the immune landscapes of 402 the IBC_Pure and IBC_LN groups, the abundance of 16 different cell types 403 was computed using $x$ Cell based on proteomic data of $s E V s$ retrieved from the 404 blood of the 105 abovementioned IBC samples (Fig 4B, Appendix Table 4). 405 We found that the enrichment scores of B cells, basophils, CD4+ T cells, CD4+ 406 naive T cells, dendritic cells (DCs), mesangial cells, activated dendritic cells 407 (aDCs), and immature dendritic cells (iDCs) were higher in the IBC_Pure group than in the IBC_LN group. On the other hand, enrichment scores for 409 adipocytes, CD8+ T cells, CD8+ naive T cells, multipotent progenitors (MPPs), 410 macrophages, megakaryocytes, platelets, and sebocytes were higher in the 411 IBC_LN group than in the IBC_Pure group (FC $>1.5$, Student's $t$ test $p<0.05)$ 412 (Fig 4B, Appendix Table 4). The enhanced adipocyte enrichment scores in 413 sEVs from IBC_LN samples attracted our attention (Fig 4B, Appendix Fig S4C, 414 Table 4). There was a positive correlation between adipogenesis and 415 adipocytes (Spearman rho $=0.188, p=5.507$ e-02) (Appendix Fig S4D). 
Adipocytes were correlated with the VEGF signaling pathway (Fig 4C), and the VEGF signaling pathway was upregulated in the IBC_LN group (Fig 4D). A previously reported comparative cytokine array analysis of adipocyte-conditioned medium (ACM) revealed the upregulation of a group of cytokines belonging to the VEGF signaling pathway in ACM (Sahoo et al, 2018).

422 The VEGF signaling pathway was correlated with MPPs (Fig 4E), which 423 were upregulated in the IBC_LN group (Appendix Fig S4E) and positively 424 correlated with the coagulation pathway (Spearman rho $=0.295$, $p$ value $=$ 2.216e-03) (Appendix Fig S4F). At the same time, platelets were positively correlated with the coagulation pathway (Spearman rho $=0.209, \mathrm{p}$ value $=$ 3.225e-02) (Appendix Fig S4G). The enrichment scores of platelets were upregulated in the IBC_LN group (Appendix Fig S4H). Experimental evidence has highlighted platelets as active players in all steps of tumorigenesis,

430 including cancer growth, cancer cell extravasation and metastasis 431 (Haemmerle et al, 2018). Many of the molecules that are highly associated 432 with platelets are angiogenesis- and metastasis-related molecules (e.g., KIF5B, 433 ARHGDIA, ARPC1B, DYNLL2, NUP98, IQGAP2, PTPRJ, PTPRF, MST1L, 434 and MMP3) (Fig 4F, Appendix Fig S4I). In addition, we found that adipocytes, 435 MPPs, and MEPs were significantly increased in the tissue samples of 40 additional IBC patients (IBC_Pure, $n=12$; IBC_LN, $n=28$ ), and the platelet

437 count in BC patients with LN metastasis $(n=43)$ was significantly higher than 438 that in BC patients without LN metastasis $(n=45)$ in our cohort (Student's $t$ 439 test, $p<0.05$ ) (Fig 4H and I, Appendix Fig S4J and K).

\section{Twelve-protein diagnostic model for LN metastasis}

442 To generate a protein signature that stratifies patients with or without LN 443 metastases, we performed random forest classification to identify a subset of 
444 sEV proteins that accurately discriminates between IBC_LN and IBC_Pure 445 samples. As before, sEV samples were evenly partitioned based on sample 446 type (i.e., IBC_LN samples vs. IBC_Pure samples), and 70\% of samples were 447 used as a training set, with the remaining 30\% used as an independent test set. 448 By comparing the IBC_LN- and IBC_Pure-derived sEV proteomes, we 449 discovered that the best partition was achieved with 12 sEV proteins (PEPD, 450 NCL, PARP1, ACTA2, ACTG2, TBCA, TTYH3, MATR3, KPNB1, KRT16, 451 RANBP2, and CCT6A). Based on this 12-protein signature, applying 5-fold 452 cross-validation to the training set yielded a sensitivity (true positive rate) of $453100 \%$ and specificity (true negative rate) of 100\% (Fig 4J and L, Appendix Fig 454 S4J). When applying the protein signature for discriminating BC patients with 455 or without LN metastasis to the independent test set samples, it had a 456 sensitivity of $81 \%$ and a specificity of $81 \%$ (Fig 4M and N, Appendix Fig S4E). 457 In addition, we used the CPTAC breast cancer dataset $(\mathrm{n}=77)$ as an external 458 validation test set and achieved $100 \%$ sensitivity and $100 \%$ specificity (Mertins 459 et al, 2016) (Fig 4K).

\section{Potential sEV survival biomarkers for distant metastases of BC}

462 To identify universal biomarkers associated with distant metastasis, we 463 performed further analysis based on the proteomic profiles of 7 ductal 464 carcinoma in situ (DCIS) samples and 21 distant metastasis (D-MET) (e.g., 465 M-Multiple $(n=5)$, M-Lung $(n=3)$, M-Liver $(n=4)$, M-Bone $(n=7)$, M-Chest 466 wall $(n=1)$, and M-Soft tissue $(n=1))$ samples in our cohort. Clustering and 467 cluster-specific enrichment analyses of the upregulated proteins using DAVID 468 (KEGG gene sets) pathway annotations clearly showed distinctive biological 469 processes and pathways enriched in D-MET samples compared to DCIS 470 samples (Fig 5A). Compared with DCIS samples, D-MET samples showed an 471 upregulation of focal adhesion (i.e., FLNA and vitronectin (VTN)) (Fisher's 
472 exact test, $p=5.45 e-03$ ), metabolism-related pathways (e.g., carbon 473 metabolism (i.e., PKM, G6PD, and TALDO1) (Fisher's exact test, $p=$ 474 5.42e-05), glycolysis/gluconeogenesis (i.e., FBP1, LDHB, and PDHB) 475 (Fisher's exact test, $p=1.33 e-02$ ), fatty acid metabolism (i.e., ACACA, 476 HSD17B12, and HACD3) (Fisher's exact test, $p=1.83 \mathrm{e}-02)$ ), and complement 477 and coagulation cascades (i.e., CPB2, alpha-1-antitrypsin (SERPINA1), CFH, 478 C7, heparin cofactor 2 (SERPIND1), F10, F12, SERPINF2, SERPINE1, F2, 479 TFPI, and KNG1) (Fisher's exact test, $p=3.58 \mathrm{e}-02)$ (Fig 5A and B). We found 480 that 24 sEV proteins were significantly overexpressed in distant metastatic 481 samples (D-MET median $/ \mathrm{DCIS}_{\text {median }}>2$-fold, Student's $t$ test, $p<0.05$ ), 482 suggesting that they may be potential serum sEV protein markers for LN 483 metastasis of $\mathrm{BC}$ (Fig 5B). Among them, $5 \mathrm{sEV}$ proteins (PDHB, FBP1, 484 PPP4C, GP1BA, and TFPI) were identified in $>75 \%$ of D-MET samples (Fig 485 5B). Remarkably, 11 sEV proteins (FLNA, VTN, PKM, PDHB, G6PD, TALDO1, 486 LDHB, ACACA, PPP4C, C7 and F2) were highly expressed in BC and were 487 associated with poor prognosis in the Tang et al. BC cohort and the Liu et al. 488 BC cohort (Liu et al, 2014; Tang et al., 2018) (Fig 5B and C, Appendix Fig 489 S5A).

Potential organ-specific sEV survival biomarkers for distant metastases of $B C$

493 Furthermore, we performed pathway enrichment analysis comparing 494 differentially expressed proteins among three different types of organ 495 metastasis samples (M-Lung, M-Liver, and M-Bone samples). M-Lung sEVs 496 showed upregulation of complement and coagulation cascades (i.e., CFD, C6, 497 and SERPING1) (Fisher's exact test, $p=1.36 \mathrm{e}-02$ ), focal adhesion (i.e., 498 ITGB3, ITGA2B, and VCL) (Fisher's exact test, $p=2.63 \mathrm{e}-02$ ), and gap 499 junctions (i.e., TUBB2B, TUBB2A, and TUBB) (Fisher's exact test, $p=$ 
500 3.49e-02) (Fig 5D, Appendix Table 5). This finding is consistent with recent 501 reports that focal adhesion and regulation of actin cytoskeleton signaling are 502 involved in lung metastases of BC (Zeng et al, 2019). Interestingly, we found 503 that abundant metabolism-related pathways were enriched in M-Liver sEVs, 504 including fatty acid metabolism (i.e., ACADVL, TECR, and ACSL5) (Fisher's 505 exact test, $p=4.56 \mathrm{e}-02$ ), galactose metabolism (i.e., GLB1, PGM5, and PGM1) 506 (Fisher's exact test, $p=1.90 \mathrm{e}-02$ ), and starch and sucrose metabolism (i.e., 507 AMY2A, AMY1A, and AMY2B) (Fisher's exact test, $p=7.99 \mathrm{e}-05$ ) (Fig 5D, 508 Appendix Table 5). M-Bone sEV samples showed upregulation of protein 509 processing in the endoplasmic reticulum (i.e., HSPH1, STT3A, RAD23A, $510 \mathrm{P} 4 \mathrm{HB}$, and SEC23B) (Fisher's exact test, $p=1.90 \mathrm{e}-02$ ) and nucleotide 511 excision repair (i.e., RPA1, RAD23A, and CUL4B) (Fisher's exact test, $p=$ 512 3.31e-02) (Fig 5D, Appendix Table 5). These results suggest that although 513 upregulated expression of adhesion, metabolism, and angiogenesis pathways 514 are common features of distant metastases, different metastases are biased. 515 M-Lung was the adhesion type, M-Liver was the metabolism type, and M-Bone was the repair type.

517 We found that GMDS was specifically highly expressed in M-Liver, P4HB 518 was specifically highly expressed in M-Bone, and C6, TUBB, SERPING1 and 519 VCL were specifically highly expressed in M-Lung (Fig 5E-G). In the Tang et al. 520 BC cohort, the high expression of C6 and VCL was associated with poor 521 prognosis, suggesting that they may be survival markers for lung metastasis of 522 BC (Tang et al., 2018) (Fig 5G and H, Appendix Fig S5B).

\section{Potential BC-derived sEV molecules govern organ-specific metastasis}

525 Metastatic organotropism has remained an enigmatic issue. A recent study 526 showed that cancer-derived sEV uptake by organ-specific cells may govern organ-specific metastasis (Hoshino et al., 2015). To examine whether sEV 
bioRxiv preprint doi: https://doi.org/10.1101/2021.1126 470104 - this version posted November 27, 2021. The copyright holder for this preprint (which was not certified by peer review) is the author/funder, who has granted bioRxiv a license to display the preprint in perpetuity. It is made available under aCC-BY-NC-ND 4.0 International license.

528 proteins may guide the colonization of BC cells in specific organs, we 529 computed the abundance of specific cell types in each of the distant metastatic 530 samples using xCell (Fig 6 A-D, Appendix Fig S6A and B, Appendix Table 6). 531 The analysis showed an enhanced enrichment score of chondrocytes in 532 M-Bone sEVs, which was 6-fold, 1.75-fold, and 2.75-fold higher than that in 533 DCIS, M-Lung, and M-Liver sEVs, respectively (Fig 6B). In contrast, the 534 enrichment score of myocytes in M-Lung sEVs was upregulated by 2.20-fold, 535 5.32-fold, and 1.82-fold compared to that in DCIS, M-Liver, and M-Bone sEVs, 536 respectively (Fig 6C). Moreover, the enrichment score of fibroblasts in M-Liver 537 samples was significantly elevated by 4.10 -fold, 12.77-fold, and 5.33-fold 538 compared to DCIS, M-Lung, and M-Bone samples, respectively (Fig 6D) 539 (Student's $t$ test, $p<0.05)$. Therefore, the organ specificity of $\mathrm{sEV}$ 540 biodistribution matched the organotropic distribution of tumor cells.

541 A previous study suggested that specific exosomal integrins were 542 associated with metastatic organotropism by dictating premetastatic niche 543 formation (Hoshino et al., 2015). In our dataset, we identified 25 integrins 544 enriched in M-Bone, M-Lung and M-Liver sEVs. Further analysis revealed that 545 ITGA1 was primarily detected in M-Bone sEVs, ITGA7 and ITGA9 were 546 abundantly enriched in M-Liver sEVs, and ITGB3 and ITGA2B were 547 abundantly enriched in M-Lung sEVs (Fig 6E, Appendix Fig S6C and D).

548 In addition to adhesive properties, sEV integrins can upregulate 549 promigratory and proinflammatory $\$ 100$ molecules, which influence 550 premetastatic niche formation (Hoshino et al., 2015). To determine the pattern 551 of sEV-S100 molecules in tumor metastasis, we identified 16 S100 molecules 552 from M-Bone, M-Lung and M-Liver sEVs. The analysis revealed that S100A8 553 was primarily detected in sEV-derived proteins from M-Bone samples (Fig 6E). 554 S100A13 was primarily detected in M-Liver samples (Appendix Fig S6C). 555 Interestingly, S100A7A was abundantly present in sEV-derived proteins from 
556 M-Lung samples (Appendix Fig S6D). In addition, we verified that ITGA1 was

557 significantly increased in M-Bone tissue samples in our additional BC cohort 558 (DCIS $(n=4)$, M-Liver $(n=4)$, M-Lung $(n=4)$, and M-Bone $(n=8))$ (Student's t 559 test, $p<0.05$ ) (Fig 6F, Appendix Fig S6A). Consistently, S100A8, S100A13, 560 and S100A7A were significantly increased in M-Bone, M-Liver, and M-Lung 561 tissue samples in our additional BC cohort (DCIS $(n=3)$, M-Liver $(n=3)$, 562 M-Lung $(\mathrm{n}=4)$, and M-Bone $(\mathrm{n}=8)$ ) (Student's $t$ test, $p<0.05)$ (Fig 6G, 563 Appendix Fig S6B, E and F). Taken together, these results suggested a 564 correlation between specific sEV integrins and S100 molecules and tissue 565 organotropism (Fig 6H).

\section{Discussion}

568 Blood tests remain the most readily accessible source for the early detection, classification, and treatment guidance of BC patients. The billions of sEVs

570 circulating in blood could represent an essential component of liquid biopsy 571 (Miyagi et al, 2021). Despite previous studies on BC-derived sEVs (Chen et al, 572 2017), there is a lack of a comprehensive understanding of BC-specific sEV 573 characteristics and their composition and consensus on unique BC biomarkers 574 due to limited sEV proteome data from human samples.

575 Here, we performed a large-scale comprehensive analysis of sEV proteomes from 167 serum samples obtained from patients with BC, patients 577 with BD, and healthy individuals. Firstly, we applied this eight-protein (STAT1, 578 PON1, APOC1, APOC2, MMP2, IGHV4-39, IGHV3-53, and ADD2) identifier to 579 sEV samples of the independent test set, the model achieved $97 \%$ sensitivity 580 and $83 \%$ specificity in the diagnosis of BC. This study may provide reference 581 value for differentiating benign and malignant breast tumors using serum in the 582 future. 
$583 \mathrm{BC}$ is a heterogeneous disease in terms of molecular alterations, cellular 584 compositions, and clinical outcomes (Wagner et al, 2019). Therefore, the classification of molecular subtype is an important tool for treatment and 586 prognosis evaluation. Clinically, based on the expression of ER, PR, Her2, and $587 \mathrm{Ki} 67$ by $\mathrm{IHC}, \mathrm{BC}$ is categorized into various molecular subtypes (Holm et al, 2021). However, the patterns of these biological indicators may change during the course of BC progression, so they may be used to adjust treatment

590 strategies accordingly (Ju et al, 2018). Thus, we speculated that an sEV-based 591 in vitro diagnostic strategy is an emerging approach complementary to tissue pathology. Unfortunately, we failed to confirm the existence of ER, PR, HER2, 593 and Ki67 in the serum sEV datasets, indicating that they may either have a low 594 abundance or be lacking in serum sEVs. However, further analyses of 595 differentially regulated sEV-derived proteins in luminal $A$, luminal $B$, 596 Her2-enriched, and TNBC samples clearly showed significant differences in 597 the proteins and biological pathways involved. By comparing proteomic 598 profiles among diverse molecular subtypes of BC, we constructed a 61-protein 599 classifier. The ROC curve derived from the 61-protein signature showed good 600 sensitivity and specificity, with an AUC of 1.0. Then, the 61-protein signature 601 was validated in the test set, resulting in a ROC curve with an AUC of 0.875 . 602 This work may provide reference value for the diagnosis of clinical subtypes of 603 BC using serum in the future.

604 An accurate preoperative assessment of $L N$ status is one of the most 605 important prognostic factors determining the long-term outcome (Banerjee et 606 al, 2004). Although noninvasive imaging modalities such as ultrasonography, 607 computed tomography, and magnetic resonance imaging have been widely 608 adopted for the clinical evaluation of LN status before surgery, the sensitivity of 609 these modalities is not satisfactory (Song, 2020). In the present study, PCA 610 demonstrated a clear distinction between IBC_LN samples and IBC_Pure 
611 samples, which further highlighted the diverse proteomic patterns between

612 IBC with or without LN metastasis. Hence, we constructed an sEV-based

613 protein signature that predicted LN metastasis at the serum sEV proteomic

614 level based on machine learning classification, showing $81 \%$ and $81 \%$

615 specificity and sensitivity, respectively. In addition, we used the CPTAC BC

616 dataset $(n=77)$ as an external validation test set and achieved $100 \%$

617 sensitivity and $100 \%$ specificity. These data suggest that tumor-associated

618 sEV proteins can serve as biomarkers for early-stage cancer detection of LN

619 metastasis.

620 Previous studies showed that adhesion and ECM molecules, such as

621 integrins, tenascin and periostin, were associated with distant metastasis of

622 disseminating cancer cells (Fukuda et al, 2015; Oskarsson et al, 2011; 623 Radisky et al, 2002; Weaver et al, 1997). Regarding the research on this 624 aspect, Hoshino et al. defined a specific repertoire of integrins expressed on 625 cancer-derived exosomes, distinct from cancer cells, that dictate metastatic 626 tropism (Hoshino et al., 2015). In our study, we identified 25 integrins 627 abundantly present in human bone-, lung- and liver-tropic metastatic sEVs by 628 quantitative mass spectrometry. Notably, we found that sEVs expressing 629 ITGA1 may specifically bind to chondrocytes, which are related to bone 630 tropism. sEVs expressing ITGB3 and ITGA2B may specifically bind to 631 lung-resident myocytes, mediating lung tropism. However, sEVs expressing 632 ITGA7 and ITGA9 may bind liver-resident fibroblasts, governing liver tropism. 633 Moreover, we revealed that the pattern of sEV-S100 molecules was correlated 634 with tissue organotropism and could serve as a biomarker for distant 635 metastasis (Fig 6H).

636 In conclusion, our findings show that proteins carried by BC-derived sEVs 637 could be used as a novel, minimally invasive liquid biopsy tool for the early 638 detection of $\mathrm{BC}$, as well as for discriminating molecular subtypes, LN 
639 involvement status, and organotropic metastasis. These findings could 640 advance the implementation of routine serum sEV-based screening in the

641 clinic.

\section{Materials and Methods}

\section{Sample collection}

645 Serum sample collection was approved by Shanghai General Hospital 646 Shanghai Jiao Tong University School of Medicine (Shanghai, China, permit 647 number [2017]KY053), and all patients provided proper consent before 648 samples were collected. Serum samples were collected between March 2011 649 and August 2019. Detailed information is shown in Appendix Table 1.

\section{sEV extraction}

652 Isolation of exosomes was performed by differential ultracentrifugation

653 following established centrifugation times and parameters (An et al, 2018; Gao 654 et al, 2021; Lakhter et al, 2018; Takov et al, 2019; Thery et al, 2006). Firstly, 1 $655 \mathrm{~mL}$ serum was thawed on ice and centrifuged at $3,000 \mathrm{~g}$ for $10 \mathrm{~min}$ at $4^{\circ} \mathrm{C}$. The 656 supernatant was removed, and large vesicles were removed with another 657 centrifugation step at $10,000 \mathrm{~g}$ for $20 \mathrm{~min}$ at $4^{\circ} \mathrm{C}$ and the supernatant was 658 diluted with $25 \mathrm{~mL}$ PBS and filtered through a $0.22 \mu \mathrm{m}$ centrifugal filter device 659 to remove any large contaminating vesicles. Secondly, filtered serum was 660 centrifuge at an overspeed of $150,000 \mathrm{~g}$ for $4 \mathrm{~h}$, the milky white floating object 661 at the top was sucked away. Thirdly, centrifuged material was resuspended 662 with $25 \mathrm{~mL}$ PBS and further centrifuged at $4^{\circ} \mathrm{C}$ for $150,000 \mathrm{~g}$ for $2 \mathrm{~h}$. Fourthly, 663 supernatant was discarded and $200 \mu \mathrm{L}$ solution was retained at the bottom to 664 resuspend the precipitate. Isolation and relative purity of the sEVs were confirmed by NTA, transmission electron microscopy (TEM) and immunoblot. 

dried by vacuum centrifugation and redissolved in 30-50 $\mu \mathrm{L}$ of $8 \mathrm{M}$ urea/50 $\mathrm{mM}$ ammonium bicarbonate/10 $\mathrm{mm}$ DTT. Following lysis and reduction,

671 proteins were alkylated using 20 or $30 \mathrm{mM}$ iodoacetamide (Sigma, St. Louis, 672 MO, USA). Proteins were digested with trypsin (Promega, Madison, WI, USA) 673 at an enzyme-to-protein mass ratio of $1: 50$ overnight at $37^{\circ} \mathrm{C}$, and peptides 674 were then extracted and dried (SpeedVac, Eppendorf). Peptides were 675 desalted and concentrated using Empore $\mathrm{C}_{18}$-based solid phase extraction 676 prior to analysis by high resolution/high mass accuracy reversed-phase $\left(\mathrm{C}_{18}\right)$ 677 nano-LC-MS/MS.

\section{Liquid chromatography}

680 We employed an EASY-nLC 1200 ultra-high-pressure system liquid 681 chromatography system (Thermo Fisher Scientific). Peptides were separated 682 within $75 \mathrm{~min}$ at a flow rate of $600 \mathrm{~nL} / \mathrm{min}$ on a $150 \mu \mathrm{m} \mathrm{I.D.} \times 15 \mathrm{~cm}$ column 683 with a laser-pulled electrospray emitter packed with $1.9 \mu \mathrm{m}$ ReproSil-Pur 120 $684 \mathrm{C}_{18} \mathrm{AQ}$ particles (Dr. Maisch). Mobile phases $\mathrm{A}$ and $\mathrm{B}$ were water and 685 acetonitrile with 0.1 vol\% FA, respectively. The \%B was linearly increased 686 from 15 to $30 \%$ within 75 min.

\section{Mass spectrometry}

689 Samples were analysed on a Q-Exactive-HF mass spectrometer (Thermo 690 Fisher Scientific) via a nanoelectrospray ion source (Thermo Fisher Scientific).

691 The mass spectrometer was operated in data-independent mode for ion 692 mobility-enhanced spectral library generation. Typically, $75 \%$ of samples were 693 injected. The peptides were dissolved in $12 \mu \mathrm{L}$ of loading buffer $(0.1 \%$ formic 
694 acid), and $9 \mu \mathrm{L}$ was loaded onto a $100 \mu \mathrm{m} \mathrm{I.D.} \times 2.5 \mathrm{~cm} \mathrm{C}_{18}$ trap column at a

695 maximum pressure of 280 bar with $14 \mu \mathrm{L}$ of solvent $A(0.1 \%$ formic acid). The

696 DIA method consisted of an MS1 scan from $300-1400 \mathrm{~m} / \mathrm{z}$ at $60 \mathrm{k}$ resolution

697 (AGC target $4 \mathrm{e} 5$ or $50 \mathrm{~ms}$ ). Then, 30 DIA segments were acquired at $15 \mathrm{k}$

698 resolution with an AGC target of $5 \mathrm{e} 4$ or $22 \mathrm{~ms}$ for maximal injection time. The

699 setting "injections for all available parallelizable time" was enabled. HCD

700 fragmentation was set to a normalized collision energy of $30 \%$. The spectra

701 were recorded in profile mode. The default charge state for the MS2 scan was

702 set to 3 .

703

704 Peptide identification and protein quantification

705 All data were processed using Firmiana (Feng et al, 2017). The DIA data were 706 searched against the UniProt human protein database using FragPipe (v.12.1) 707 with MSFragger (2.2) (Kong et al, 2017). The mass tolerances were 20 ppm 708 for precursor and 50 mmu for product ions. Up to two missed cleavages were 709 allowed. The search engine set cysteine carbamidomethylation as a fixed 710 modification and $\mathrm{N}$-acetylation and oxidation of methionine as variable

711 modifications. Precursor ion score charges were limited to $+2,+3$, and +4 . The 712 data were also searched against a decoy database so that protein 713 identifications were accepted at a false discovery rate (FDR) of $5 \%$.

714 The DIA data was analysed using DIANN (v.1.7.0) (Demichev et al, 2020).

715 The quantification of identified peptides was calculated as the average 716 chromatographic fragment ion peak areas across all reference spectra 717 libraries. Label-free protein quantifications were calculated using a label-free, 718 intensity-based absolute quantification (iBAQ) approach (Zhang et al, 2012).

719 We calculated the peak area values as parts of the corresponding proteins. 720 The fraction of total (FOT) was used to represent the normalized abundance of 721 a particular protein across samples. FOT was defined as a protein's iBAQ 
722 divided by the total $\mathrm{BBAQ}$ of all identified proteins within a sample. The FOT

723 values were multiplied by $10^{5}$ for ease of presentation, and missing values

724 were imputed to $10^{-5}$. The raw proteomics data files are hosted by iProX and

725 can be accessed at https://www.iprox.cn (Project ID: IPX0003429000).

\section{Statistical analysis}

728 To impute the proteomic data, we first screened more than $50 \%$ of the 729 identified proteins in each group and divided the data into two parts. When the 730 protein detection rate was $<50 \%$, the missing value was replaced with one 731 tenth of the minimum value. For these proteins, no imputation was applied.

732 When the protein detection rate was $>0.5$, the missing value was probably due

733 to the detection accuracy limitation of LC/MS. In this case, we first calculated 734 the missing probability of a protein using the R package "impute" 735 (https://git.bioconductor.org/ packages/impute) based on the K-NN algorithm.

736 Meta-analysis-based discovery and validation of survival biomarkers was 737 carried out using Kaplan-Meier Plotter (http://kmplot.com/analysis/).

\section{Principal component analysis (PCA)}

740 The imputed data were then normalized using the LogNorm algorithm. The 741 PCA function of the $R$ package "factoextra"

742 (https://cran.r-project.org/web/packages/ factoextra /index.html) was used to

743 implement unsupervised clustering analysis. The 95\% confidence coverage 744 was represented by a coloured ellipse for each group and was calculated 745 based on the mean and covariance of points in the different groups. 1,734, 746 1,038, and 1,116 proteins (features) were used for PCA to illustrate the global 747 proteomic differences among BC $(n=126)$, BD $(n=17)$ and HD $(n=24)$, the 748 global proteomic differences among the luminal $A(n=20)$, luminal $B(n=50)$, 749 Her2-enriched $(\mathrm{n}=21)$, and triple-negative breast cancer (TNBC) $(\mathrm{n}=23)$ 
750 subtypes, and the global proteomic differences between IBC_Pure $(n=54)$

751 and IBC_LN $(\mathrm{n}=51)$ (Appendix Fig S2A, 3A, 4A).

\section{Global Heatmap}

754 Each gene expression value in the global proteomic expression matrix was 755 transformed to a z-score across all the samples. The z-score-transformed 756 matrix was clustered using the $\mathrm{R}$ package "pheatmap"

757 (https://cran.r-project.org/web/packages/pheatmap/index.html).

\section{Pathway enrichment analysis}

760 Pathway enrichment analysis was performed by DAVID (https://david.

761 ncifcrf.gov) and ConsensusPathDB (http://cpdb. molgen.mpg.de), and

762 significance in the pathway enrichment analysis was determined by Fisher's 763 exact test on the basis of Kyoto Encyclopedia of Genes and Genomes (KEGG) 764 pathways and categorical annotations, including Gene Ontology (GO) 765 biological process (GOBP) terms and Reactome (https://reactome.org).

Multiplex immunohistochemistry (mIHC) with tyramide signal 768 amplification

769 Tissues or cells were prepared for detection with kits using standard fixation 770 and embedding techniques. Each slide was baked in an oven at $65^{\circ} \mathrm{C}$ for $1 \mathrm{~h}$,

771 dewaxed with xylene $(3 \times 10 \mathrm{~min})$ and rehydrated through a graded series of 772 ethanol solutions ( $100 \%$ ethanol, $95 \%$ ethanol, $75 \%$ ethanol, $50 \%$ ethanol)and 773 each step took $5 \mathrm{~min}$. After rehydration, immersing the slides in the boiled 774 appropriate AR buffer, and placed in a microwave for $15 \mathrm{~min}$ at $20 \%$ power. 775 After naturally cooling to room temperature, washing the slides with TBST. 776 Then we used blocking buffer to incubate tissue section for $10 \mathrm{~min}$. The 777 blocking buffer was drained, and Primary Antibody Working Solution was 
778 applied. CD45RA (1:3000; ab755; Abcam), CD34 (1:6000; ab81289; Abcam),

779 CD38 (1:800; ab108403; Abcam), CD71 (1:800; ab214039; Abcam), and

780 CDH1 (1:10000; ab181860; Abcam) were used. The slides were incubated at $7814^{\circ} \mathrm{C}$ overnight or at room temperature for $1 \mathrm{~h}$; the time may be adjusted 782 according to different characteristics of the antibody. After washing the slides 783 with TBST, incubate them in polymer HRP Ms+Rb for $15 \mathrm{~min}$ at room 784 temperature. Washing the slides twice again. Working Solution $(100-300 \mu \mathrm{L})$ 785 was pipetted onto each slide at room temperature for $10 \mathrm{~min}$. And then 786 immersed in the appropriate AR buffer. This microwave step strips the 787 primary-secondary-HRP complex, allowing the introduction of the next primary 788 antibody. For detection of the next target with fluorophores, we restarted the 789 protocol at blocking. Once all 5 targets were labelled, Opal Polaris 780 790 labelling was continued.

791 Dropping TSA-DIG Working Solution onto slides and incubating at room 792 temperature for $10 \mathrm{~min}$. Repeat the previous microwave repair steps after 793 washing the slides. Polaris 780 Working Solution was pipetted onto each slide 794 and incubated at room temperature for $1 \mathrm{~h}$. DAPI working solution was applied 795 for 5 min. The slides were washed twice again. After the slides were slightly 796 dry, a super quench sealing tablet was added to the slides with a pipette, and 797 the sample area was immersed.

799 Immunohistochemistry (IHC)

800 Firstly, the sections were baked at $65^{\circ} \mathrm{C}$ for $1 \mathrm{~h}$ and incubated in xylene three 801 times for 10 min each time. Then, the sections were hydrated by a graded 802 series of ethanol (100\% ethanol, $95 \%$ ethanol, $75 \%$ ethanol, $50 \%$ ethanol and $803 \mathrm{ddH}_{2} \mathrm{O}$ ), and each step took $5 \mathrm{~min}$. Antigen retrieval was conducted using a 804 microwave oven: 3 min at $100 \%$ power and 15 min at 20\% power filled with 10 $\mathrm{mM}$ sodium citrate buffer ( $\mathrm{pH} 6.0$ ). After naturally cooling to room temperature 
806 and washed in $\mathrm{ddH}_{2} \mathrm{O}$, we blocked the sections with $5 \%$ normal goat serum for $80710 \mathrm{~min}$, incubated sections in $3 \% \mathrm{H}_{2} \mathrm{O}_{2}$ for 10 min at room temperature, and 808 washed the sections twice in PBS for $5 \mathrm{~min}$. The following antibodies were 809 diluted in the appropriate concentrations: PPARg (1:10000; ab59256; Abcam), 810 S100A8 (1:800; 15792-1-AP; Proteintech), S100A13 (1:1200; ab109252; 811 Abcam), S100A7A (1:400; DF8517; Affinity), and ITGA1 (1:300; 22146-1 AP; 812 Proteintech). These antibodies were incubated with the sections overnight at $8134^{\circ} \mathrm{C}$

814 The next day, after washing the sections twice in PBS, we used an IHC Kit 815 (ZSGB-BIO, Beijing, China, Cat\# SP-9000), incubated the sections with 816 biotin-labelled secondary antibody for $15 \mathrm{~min}$. After washing sections twice in 817 PBS, incubating the sections with horseradish enzyme-labelled Streptomyces 818 ovalbumin working solution for $15 \mathrm{~min}$. Finally, We used DAB solution to stain 819 the tissues. Then, using haematoxylin to stain nuclears and washing them in $820 \mathrm{ddH}_{2} \mathrm{O}$. Finally, the sections were dehydrated by graded ethanol ( $50 \%$ ethanol, $82175 \%$ ethanol, $95 \%$ ethanol, and $100 \%$ ethanol). We dried the slides in a fume cupboard for at least 20 min and mounted coverslips.

\section{Acknowledgments}

825 This work was supported by the National Natural Science Funds (grant numbers 82073269, 81772802 and M-0349), Shanghai Science and

827 Technology Innovation Action Plan (grant number 20XD1402800), Clinical 828 Research Plan of SHDC (grant number SHDC2020CR2065B), Clinical 829 Research Innovation Plan of Shanghai General Hospital (grant number 830 CTCCR-2016B05), National Key R\&D Program of China (grant numbers 831 2017YFA0505102, 2016YFA0502500, 2018YFA0507501, and 832 2017YFC0908404), National Natural Science Foundation of China (grant 833 numbers 31770886, 1972933, and 31700682), Science and Technology 
bioRxiv preprint doi: https://doi.org/10.1101/2021.11.26.470104; this version posted November 27, 2021. The copyright holder for this preprint (which was not certified by peer review) is the author/funder, who has granted bioRxiv a license to display the preprint in perpetuity. It is made available under aCC-BY-NC-ND 4.0 International license.

834 Commission of Shanghai Municipality (grant number 2017SHZDZX01), Major 835 Project of Special Development Funds of Zhangjiang National Independent 836 Innovation Demonstration Zone (grant number ZJ2019-ZD-004).

\section{Author Contributions}

839 H.X.W. contributed to idea, conception, and study design. H.X.W., C.D., and H.W.Z. wrote the paper and supervised the project. G.F.X., S.Q.D. and M.J.H. conducted the mass spectrometry analysis. W.Y.H. and the other authors carried out all the remaining experiments. All authors discussed the results, commented on the project and approved the manuscript.

\section{References}

846 An M, Wu J, Zhu J, Lubman DM (2018) Comparison of an Optimized 847 Ultracentrifugation Method versus Size-Exclusion Chromatography for 848 Isolation of Exosomes from Human Serum. J Proteome Res 17: 3599-3605

849 Aran D, Hu Z, Butte AJ (2017) xCell: digitally portraying the tissue cellular 850 heterogeneity landscape. Genome Biol 18: 220

851 Balaj L, Lessard R, Dai L, Cho YJ, Pomeroy SL, Breakefield XO, Skog J (2011) 852 Tumour microvesicles contain retrotransposon elements and amplified 853 oncogene sequences. Nat Commun 2: 180

854 Banerjee M, George J, Song EY, Roy A, Hryniuk W (2004) Tree-based model 855 for breast cancer prognostication. J Clin Oncol 22: 2567-2575

856 Becker M, Weinberger T, Chandy A, Schmukler S (2016) Depression During 857 Pregnancy and Postpartum. Curr Psychiatry Rep 18: 32

858 Cao Y (2019) Adipocyte and lipid metabolism in cancer drug resistance. J Clin 859 Invest 129: 3006-3017

860 Cassetta L, Pollard JW (2017) Repolarizing macrophages improves breast 861 cancer therapy. Cell Res 27: 963-964

862 Chen G, Huang AC, Zhang W, Zhang G, Wu M, Xu W, Yu Z, Yang J, Wang B, 863 Sun $\mathrm{H}$ et al (2018) Exosomal PD-L1 contributes to immunosuppression and is 864 associated with anti-PD-1 response. Nature 560: 382-386

865 Chen IH, Xue L, Hsu CC, Paez JS, Pan L, Andaluz H, Wendt MK, Iliuk AB, Zhu 866 JK, Tao WA (2017) Phosphoproteins in extracellular vesicles as candidate 867 markers for breast cancer. Proc Natl Acad Sci U S A 114: 3175-3180 
868

Choi Y, Park U, Koo HJ, Park JS, Lee DH, Kim K, Choi J (2021) Exosome-mediated diagnosis of pancreatic cancer using lectin-conjugated nanoparticles bound to selective glycans. Biosens Bioelectron 177: 112980 Colombo M, Raposo G, Thery C (2014) Biogenesis, secretion, and intercellular interactions of exosomes and other extracellular vesicles. Annu Rev Cell Dev Biol 30: 255-289

Costa-Silva B, Aiello NM, Ocean AJ, Singh S, Zhang H, Thakur BK, Becker A, Hoshino A, Mark MT, Molina $\mathrm{H}$ et al (2015) Pancreatic cancer exosomes initiate pre-metastatic niche formation in the liver. Nat Cell Biol 17: 816-826

Demichev V, Messner CB, Vernardis SI, Lilley KS, Ralser M (2020) DIA-NN: neural networks and interference correction enable deep proteome coverage in high throughput. Nat Methods 17: 41-44

Fang X, Duan Y, Adkins GB, Pan S, Wang H, Liu Y, Zhong W (2018) Highly Efficient Exosome Isolation and Protein Analysis by an Integrated Nanomaterial-Based Platform. Anal Chem 90: 2787-2795

Feng J, Ding C, Qiu N, Ni X, Zhan D, Liu W, Xia X, Li P, Lu B, Zhao Q et al (2017) Firmiana: towards a one-stop proteomic cloud platform for data processing and analysis. Nat Biotechnol 35: 409-412

Fukuda K, Sugihara E, Ohta S, Izuhara K, Funakoshi T, Amagai M, Saya H (2015) Periostin Is a Key Niche Component for Wound Metastasis of Melanoma. PLoS One 10: e0129704

Gao Y, Chen Y, Wang L, Li C, Ge W (2021) Serum-derived extracellular vesicles inhibit osteoclastogenesis in active-phase patients with SAPHO syndrome. Ther Adv Musculoskelet Dis 13: 1759720X211006966 Haemmerle M, Stone RL, Menter DG, Afshar-Kharghan V, Sood AK (2018) The Platelet Lifeline to Cancer: Challenges and Opportunities. Cancer Cell 33: 965-983

Holm J, Yu NY, Johansson A, Ploner A, Hall P, Lindstrom LS, Czene K (2021) Concordance of Immunohistochemistry-Based and Gene Expression-Based Subtyping in Breast Cancer. JNCI Cancer Spectr 5: pkaa087

Hoshino A, Costa-Silva B, Shen TL, Rodrigues G, Hashimoto A, Tesic Mark M, Molina H, Kohsaka S, Di Giannatale A, Ceder S et al (2015) Tumour exosome integrins determine organotropic metastasis. Nature 527: 329-335 Hoshino A, Kim HS, Bojmar L, Gyan KE, Cioffi M, Hernandez J, Zambirinis CP, Rodrigues G, Molina H, Heissel S et al (2020) Extracellular Vesicle and Particle Biomarkers Define Multiple Human Cancers. Cell 182: 1044-1061 e1018

Huber V, Fais S, lero M, Lugini L, Canese P, Squarcina P, Zaccheddu A, Colone M, Arancia G, Gentile M et al (2005) Human colorectal cancer cells induce T-cell death through release of proapoptotic microvesicles: role in immune escape. Gastroenterology 128: 1796-1804 
909 Johnstone RM, Adam M, Hammond JR, Orr L, Turbide C (1987) Vesicle

910 formation during reticulocyte maturation. Association of plasma membrane

911 activities with released vesicles (exosomes). J Biol Chem 262: 9412-9420

912 Ju G, Zhu R, Zhao H, Ye F, Zhang L, Lin C, Lu Y, Zhang X, Li N, Xue P et al

913 (2018) The discordance pattern of molecular sub-types between primary and

914 metastatic sites in Chinese breast cancer patients. Int $J$ Clin Exp Pathol 11:

$915 \quad$ 5938-5947

916 Kalra H, Simpson RJ, Ji H, Aikawa E, Altevogt P, Askenase P, Bond VC, 917 Borras FE, Breakefield X, Budnik V et al (2012) Vesiclepedia: a compendium

918 for extracellular vesicles with continuous community annotation. PLoS Biol 10:

919 e1001450

920 Kim DK, Kang B, Kim OY, Choi DS, Lee J, Kim SR, Go G, Yoon YJ, Kim JH, 921 Jang SC et al (2013) EVpedia: an integrated database of high-throughput data 922 for systemic analyses of extracellular vesicles. J Extracell Vesicles 2

923 Kim JS, Chang JW, Park JK, Hwang SG (2012) Increased aldehyde reductase 924 expression mediates acquired radioresistance of laryngeal cancer cells via 925 modulating p53. Cancer Biol Ther 13: 638-646

926 Kong AT, Leprevost FV, Avtonomov DM, Mellacheruvu D, Nesvizhskii AI 927 (2017) MSFragger: ultrafast and comprehensive peptide identification in mass 928 spectrometry-based proteomics. Nat Methods 14: 513-520

929 Kralj-Iglic V (2012) Stability of membranous nanostructures: a possible key 930 mechanism in cancer progression. Int J Nanomedicine 7: 3579-3596

931 Lakhter AJ, Pratt RE, Moore RE, Doucette KK, Maier BF, DiMeglio LA, Sims 932 EK (2018) Beta cell extracellular vesicle miR-21-5p cargo is increased in 933 response to inflammatory cytokines and serves as a biomarker of type 1 934 diabetes. Diabetologia 61: 1124-1134

935 Laudato S, Patil N, Abba ML, Leupold JH, Benner A, Gaiser T, Marx A, 936 Allgayer $\mathrm{H}$ (2017) P53-induced miR-30e-5p inhibits colorectal cancer invasion 937 and metastasis by targeting ITGA6 and ITGB1. Int J Cancer 141: 1879-1890 938 Lee J, Kwon MH, Kim JA, Rhee WJ (2018) Detection of exosome miRNAs 939 using molecular beacons for diagnosing prostate cancer. Artif Cells Nanomed 940 Biotechnol 46: S52-S63

941 Li A, Zhang T, Zheng M, Liu Y, Chen Z (2017a) Exosomal proteins as potential 942 markers of tumor diagnosis. J Hematol Oncol 10: 175

943 Li H, Gao C, Zhuang J, Liu L, Yang J, Liu C, Zhou C, Feng F, Liu R, Sun C 944 (2021) An mRNA characterization model predicting survival in patients with 945 invasive breast cancer based on The Cancer Genome Atlas database. Cancer 946 Biomark 30: 417-428

947 Li W, Li C, Zhou T, Liu X, Liu X, Li X, Chen D (2017b) Role of exosomal 948 proteins in cancer diagnosis. Mol Cancer 16: 145

949 Liu NQ, Stingl C, Look MP, Smid M, Braakman RB, De Marchi T, Sieuwerts 950 AM, Span PN, Sweep FC, Linderholm BK et al (2014) Comparative proteome 
951

952

953

954

955

956

957

958

959

960

961

962

963

964

965

966

967

968

969

970

971

972

973

974

975

976

977

978

979

980

981

982

983

984

985

986

987

988

989

990

991 analysis revealing an 11-protein signature for aggressive triple-negative breast cancer. J Natl Cancer Inst 106: djt376

Liu X, Li J, Cadilha BL, Markota A, Voigt C, Huang Z, Lin PP, Wang DD, Dai J, Kranz G et al (2019a) Epithelial-type systemic breast carcinoma cells with a restricted mesenchymal transition are a major source of metastasis. Sci Adv 5: eaav4275

Liu Y, Wang H, Wang X, Liu J, Li J, Wang X, Zhang Y, Bai Z, Zhou Q, Wu Y et al (2021) Prevalence and reclassification of BRCA1 and BRCA2 variants in a large, unselected Chinese Han breast cancer cohort. J Hematol Oncol 14: 18 Liu Z, Wang Y, Wang L, Yao B, Sun L, Liu R, Chen T, Niu Y, Tu K, Liu Q (2019b) Long non-coding RNA AGAP2-AS1, functioning as a competitive endogenous RNA, upregulates ANXA11 expression by sponging miR-16-5p and promotes proliferation and metastasis in hepatocellular carcinoma. $J$ Exp Clin Cancer Res 38: 194

Maas SLN, Breakefield XO, Weaver AM (2017) Extracellular Vesicles: Unique Intercellular Delivery Vehicles. Trends Cell Biol 27: 172-188

Mertins P, Mani DR, Ruggles KV, Gillette MA, Clauser KR, Wang P, Wang X, Qiao JW, Cao S, Petralia F et al (2016) Proteogenomics connects somatic mutations to signalling in breast cancer. Nature 534: 55-62

Milane L, Singh A, Mattheolabakis G, Suresh M, Amiji MM (2015) Exosome mediated communication within the tumor microenvironment. $J$ Control Release 219: 278-294

Miyagi T, Miyata S, Tagami K, Hiratsuka Y, Sato M, Takeda I, Kohata K, Satake N, Shimokawa H, Inoue A (2021) Prognostic model for patients with advanced cancer using a combination of routine blood test values. Support Care Cancer

Oskarsson T, Acharyya S, Zhang XH, Vanharanta S, Tavazoie SF, Morris PG, Downey RJ, Manova-Todorova K, Brogi E, Massague J (2011) Breast cancer cells produce tenascin $C$ as a metastatic niche component to colonize the lungs. Nat Med 17: 867-874

Ozer T, Geiss BJ, Henry CS (2020) Review-Chemical and Biological Sensors for Viral Detection. J Electrochem Soc 167: 037523

Peinado H, Aleckovic M, Lavotshkin S, Matei I, Costa-Silva B, Moreno-Bueno G, Hergueta-Redondo M, Williams C, Garcia-Santos G, Ghajar C et al (2012) Melanoma exosomes educate bone marrow progenitor cells toward a pro-metastatic phenotype through MET. Nat Med 18: 883-891

Peinado H, Lavotshkin S, Lyden D (2011) The secreted factors responsible for pre-metastatic niche formation: old sayings and new thoughts. Semin Cancer Biol 21: 139-146

Peng J, Chen J, Xie F, Bao W, Xu H, Wang H, Xu Y, Du Z (2019) Herceptin-conjugated paclitaxel loaded PCL-PEG worm-like nanocrystal 
992 micelles for the combinatorial treatment of HER2-positive breast cancer.

993 Biomaterials 222: 119420

994 Radisky D, Muschler J, Bissell MJ (2002) Order and disorder: the role of

995 extracellular matrix in epithelial cancer. Cancer Invest 20: 139-153

996 Raposo G, Stoorvogel W (2013) Extracellular vesicles: exosomes,

997 microvesicles, and friends. J Cell Biol 200: 373-383

998 Rontogianni S, Synadaki E, Li B, Liefaard MC, Lips EH, Wesseling J, Wu W,

999 Altelaar M (2019) Proteomic profiling of extracellular vesicles allows for human

1000 breast cancer subtyping. Commun Biol 2: 325

1001 Sahoo SS, Lombard JM, lus Y, O'Sullivan R, Wood LG, Nahar P, Jaaback K, 1002 Tanwar PS (2018) Adipose-Derived VEGF-mTOR Signaling Promotes

1003 Endometrial Hyperplasia and Cancer: Implications for Obese Women. Mol 1004 Cancer Res 16: 309-321

1005 Siegel RL, Miller KD, Fuchs HE, Jemal A (2021) Cancer Statistics, 2021. CA: a 1006 cancer journal for clinicians 71: 7-33

1007 Skog J, Wurdinger T, van Rijn S, Meijer DH, Gainche L, Sena-Esteves M, 1008 Curry WT, Jr., Carter BS, Krichevsky AM, Breakefield XO (2008) Glioblastoma 1009 microvesicles transport RNA and proteins that promote tumour growth and 1010 provide diagnostic biomarkers. Nat Cell Biol 10: 1470-1476

1011 Song BI (2020) Nomogram using F-18 fluorodeoxyglucose positron emission 1012 tomography/computed tomography for preoperative prediction of lymph node 1013 metastasis in gastric cancer. World J Gastrointest Oncol 12: 447-456

1014 Takov K, Yellon DM, Davidson SM (2019) Comparison of small extracellular 1015 vesicles isolated from plasma by ultracentrifugation or size-exclusion 1016 chromatography: yield, purity and functional potential. J Extracell Vesicles 8: 10171560809

1018 Tang W, Zhou M, Dorsey TH, Prieto DA, Wang XW, Ruppin E, Veenstra TD, 1019 Ambs S (2018) Integrated proteotranscriptomics of breast cancer reveals 1020 globally increased protein-mRNA concordance associated with subtypes and 1021 survival. Genome Med 10: 94

1022 Thakur BK, Zhang H, Becker A, Matei I, Huang Y, Costa-Silva B, Zheng Y, 1023 Hoshino A, Brazier H, Xiang J et al (2014) Double-stranded DNA in exosomes:

1024 a novel biomarker in cancer detection. Cell Res 24: 766-769

1025 Thery C, Amigorena S, Raposo G, Clayton A (2006) Isolation and 1026 characterization of exosomes from cell culture supernatants and biological 1027 fluids. Curr Protoc Cell Biol Chapter 3: Unit 322

1028 Thery C, Ostrowski M, Segura E (2009) Membrane vesicles as conveyors of 1029 immune responses. Nat Rev Immunol 9: 581-593

1030 Valadi H, Ekstrom K, Bossios A, Sjostrand M, Lee JJ, Lotvall JO (2007) 1031 Exosome-mediated transfer of mRNAs and microRNAs is a novel mechanism 1032 of genetic exchange between cells. Nat Cell Biol 9: 654-659 
1033 Vallejos CS, Gomez HL, Cruz WR, Pinto JA, Dyer RR, Velarde R, Suazo JF,

1034 Neciosup SP, Leon M, de la Cruz MA et al (2010) Breast cancer classification

1035

1036

1037

1038

1039

1040

1041

1042

1043

1044

1045

1046

1047

1048

1049

1050

1051

1052

1053

1054

1055

1056

1057

1058

1059

1060

1061

1062

1063

1064

1065

1066

1067

1068

1069

1070

1071

1072

1073 according to immunohistochemistry markers: subtypes and association with clinicopathologic variables in a peruvian hospital database. Clin Breast Cancer 10: 294-300

Wagner J, Rapsomaniki MA, Chevrier S, Anzeneder T, Langwieder C, Dykgers A, Rees M, Ramaswamy A, Muenst S, Soysal SD et al (2019) A Single-Cell Atlas of the Tumor and Immune Ecosystem of Human Breast Cancer. Cell 177: 1330-1345 e1318

Wan JCM, Massie C, Garcia-Corbacho J, Mouliere F, Brenton JD, Caldas C, Pacey S, Baird R, Rosenfeld N (2017) Liquid biopsies come of age: towards implementation of circulating tumour DNA. Nat Rev Cancer 17: 223-238

Wang $\mathrm{H}$, Song X, Huang Q, Xu T, Yun D, Wang Y, Hu L, Yan Y, Chen H, Lu D et al (2019a) LGALS3 Promotes Treatment Resistance in Glioblastoma and Is Associated with Tumor Risk and Prognosis. Cancer Epidemiol Biomarkers Prev 28: 760-769

Wang HX, Gires O (2019) Tumor-derived extracellular vesicles in breast cancer: From bench to bedside. Cancer Lett 460: 54-64

Wang J, Liu Z, Zhang S, Wang X, Bai H, Xie M, Dong F, Ema H (2019b) Lineage marker expression on mouse hematopoietic stem cells. Exp Hematol 76: 13-23 e12

Wang M, Ji S, Shao G, Zhang J, Zhao K, Wang Z, Wu A (2018) Effect of exosome biomarkers for diagnosis and prognosis of breast cancer patients. Clin Trans/ Oncol 20: 906-911

Wang YT, Shi T, Srivastava S, Kagan J, Liu T, Rodland KD (2020) Proteomic Analysis of Exosomes for Discovery of Protein Biomarkers for Prostate and Bladder Cancer. Cancers (Basel) 12

Weaver VM, Petersen OW, Wang F, Larabell CA, Briand P, Damsky C, Bissell MJ (1997) Reversion of the malignant phenotype of human breast cells in three-dimensional culture and in vivo by integrin blocking antibodies. $J$ Cell Biol 137: 231-245

Wu D, Yan J, Shen X, Sun Y, Thulin M, Cai Y, Wik L, Shen Q, Oelrich J, Qian $X$ et al (2019) Profiling surface proteins on individual exosomes using a proximity barcoding assay. Nat Commun 10: 3854

Xavier-Ferrucio J, Scanlon V, Li X, Zhang PX, Lozovatsky L, Ayala-Lopez N, Tebaldi T, Halene S, Cao C, Fleming MD et al (2019) Low iron promotes megakaryocytic commitment of megakaryocytic-erythroid progenitors in humans and mice. Blood 134: 1547-1557

Xu R, Greening DW, Zhu HJ, Takahashi N, Simpson RJ (2016) Extracellular vesicle isolation and characterization: toward clinical application. J Clin Invest 126: $1152-1162$ 
1074 Yanez-Mo M, Siljander PR, Andreu Z, Zavec AB, Borras FE, Buzas El, Buzas K, Casal E, Cappello F, Carvalho J et al (2015) Biological properties of extracellular vesicles and their physiological functions. J Extracell Vesicles 4: 27066

1078 Yin ZQ, Liu JJ, Xu YC, Yu J, Ding GH, Yang F, Tang L, Liu BH, Ma Y, Xia YW et al (2014) A 41-gene signature derived from breast cancer stem cells as a predictor of survival. J Exp Clin Cancer Res 33: 49 Yoneda K, Imanishi N, Ichiki Y, Tanaka F (2019) A liquid biopsy in primary lung cancer. Surg Today 49: 1-14 al (2019) SEPT9_i1 regulates human breast cancer cell motility through cytoskeletal and RhoA/FAK signaling pathway regulation. Cell Death Dis 10: 720 label-free fast quantitative analysis tool for high-resolution LC-MS/MS proteomics data. Proteomics 12: 3475-3484

1090 Zhang Y, Wang XF (2015) A niche role for cancer exosomes in metastasis.

$1091 \quad$ Nat Cell Biol 17: 709-711

1092 Zhu R, Gires O, Zhu L, Liu J, Li J, Yang H, Ju G, Huang J, Ge W, Chen Y et al

1093 (2019) TSPAN8 promotes cancer cell stemness via activation of sonic Hedgehog signaling. Nat Commun 10: 2863 


\section{Figure Legends}

1097 Figure 1. Overview of the proteomic characterization of breast cancer 1098 sEVs.

1099 A Overview of the experimental design and the number of samples for

1100 proteomics analyses.

1101 B Clinical parameters are indicated in the heatmap.

1102 C Schematic diagram of the extraction process of serum-derived sEVs.

1103 D Representative TEM images of purified EVs. Scale bar-100 nm.

1104 E Immunoblots showing the expression levels of ALIX (PDCD6IP), CD63, CD9, 1105 and TSG101 in the purified EVs.

1106 F Cumulative number of protein identifications. Red denotes BC samples $(\mathrm{n}=$ 1107 126), yellow denotes BD samples $(n=17)$, and blue denotes HD samples ( $n$ $1108=24)$.

$1109 \mathrm{G}$ The number of proteins identified in 167 samples. Red denotes $B C$ samples $1110 \quad(n=126)$, yellow denotes BD samples $(n=17)$, and blue denotes HD 1111 samples $(n=24)$.

$1112 \mathrm{H}$ Venn diagram depicting the numbers of proteins detected in BC-, BD-, and 1113 HD-sEVs.

Figure 2. Proteomics features of BC-, BD- and HD-derived sEVs.

1116 A The bubble plot indicates the distinctive biological pathways of $B C, B D$, and

1117 HD. Red box, BC; yellow box, BD; blue box, HD. See Table S2.

1118 B Differentially expressed proteins in the distinctive biological pathways of BC,

$1119 \mathrm{BD}$, and HD. Fold change $>2$ and one-way ANOVA $p<0.05$.

1120 C Two proteins (MMP2 and TYMP) differentially expressed in BC, BD, and HD 1121 ( $p$ value from Student's $t$ test) and their association with clinical outcomes in $1122 \quad B C(2018$, Tang et al., $B C$ cohort, $n=118)$ ( $p$ value from log rank test). 
1123 D sEV DAMP molecules were enriched in $\mathrm{BC}$ and found in $>50 \%$ of $\mathrm{BC}$

1124 samples, with $>$ 2-fold difference and one-way ANOVA $p<0.05$

1125 E DAMP molecules enriched in BC-sEVs were significantly associated with

1126 clinical outcomes in BC (2018, Tang et al., BC cohort, $\mathrm{n}=118)$ ( $p$ value from

$1127 \log$ rank test).

1128 F Distinctive tumour microenvironment in BC. See Table S2.

1129 G Functions of BC-derived sEVs. These sEVs impact the tumour

1130 microenvironment by promoting tumour cell growth and progression,

1131 modulating immune responses, regulating angiogenesis and inducing

1132 metastatic behaviour through MEPs, endothelial cells, and Mv endothelial

$1133 \quad$ cells.

$1134 \mathrm{H}$ Classification error matrix of the training set $(70 \%)$ and test set $(30 \%)$ for the

11358 proteins using the random forest classifier. The number of samples 1136 identified is noted in each box.

1137 I Proteins with the highest predictive values in classifying BC, BD and HD 1138 samples by XGBoost.

1140 Figure 3. Proteomic landscapes of four clinical subtypes of BC-derived 1141 sEVs.

1142 A Differentially expressed proteins in luminal A, luminal B, Her2-enriched, and 1143 TNBC samples and found in $>50 \%$ of the corresponding samples, with > $1144 \quad 2$-fold difference from the other three subtypes.

1145 B Gene Ontology biological processes (GOBPs) revealed pathways that were 1146 significantly enriched in luminal A, luminal B, Her2-enriched and TNBC 1147 samples (Fisher's exact test, $p<0.05$ ). See Table S3.

1148 C Differentially expressed proteins in luminal A, luminal B, Her2-enriched, and 1149 TNBC samples. See Table S3. 
1150 D Classification error matrix of the training set $(70 \%)$ and test set $(30 \%)$ for the

115161 proteins using the XGBoost classifier. The number of samples identified

1152 is noted in each box. The bar chart above represents the predictive 1153 specificity of each subtype. The bar chart on the right represents the 1154 predictive sensitivity of each subtype.

1155 Figure 4. Potential prognostic biomarkers for IBC patients with Iymph 1156 node metastases.

1157 A GSEA of the proteomic data of 105 breast cancer samples revealed that 1158 adipogenesis was significantly upregulated in IBC_LN. IBC_LN: IBC 1159 patients with lymph node metastases.

1160 B Distinctive tumour microenvironment between IBC_Pure and IBC_LN. 1161 IBC_Pure: IBC patients without lymph node metastases; IBC_LN: IBC 1162 patients with lymph node metastases. See Table S4.

1163 C Correlation between adipocytes and the pathway of positive regulation of

1164 VEGF production. Spearman rho $=0.412$, Wilcoxon rank sum test, $p=$ $1165 \quad 1.242 \mathrm{e}-05$.

1166 D Comparison of the scores of positive regulation of VEGF production 1167 between IBC_LN and IBC_Pure. The $p$ value was calculated by the 1168 Wilcoxon rank sum test. The line and box represent median and upper and 1169 lower quartiles, respectively.

1170 E Correlation between the pathway of positive regulation of VEGF production 1171 and MPPs. Correlation coefficients and $p$ values were calculated by the 1172 Spearman correlation method.

1173 F Molecules highly associated with platelets were expressed in IBC_Pure and 1174 IBC_LN.

1175 G The pattern diagram shows the process by which adipocytes activate MPPs 1176 to generate MEPs and MKs through positive regulation of VEGF production 
1177 and finally produce platelets. The produced platelets helped breast cancer

1178 cells migrate to the lymph nodes.

$1179 \mathrm{H}$ Representative immunohistochemical images of adipocytes labelled with 1180 PPRGg. Images revealed that adipocytes prolifically grew in lymph node 1181 metastases of BC compared to primary breast cancer.

1182 I Representative fluorescence microscopy images of MPPs labelled with 1183 CD45RA (green), CD34 (red), and CD38 (yellow). Images revealed the

1184 presence of MPPs in lymph node metastases of BC, which were rare in 1185 normal lymph nodes and primary breast cancer.

$1186 \mathrm{~J}$ Classification error matrix of the training set (70\%) and test set $(30 \%)$ for the 118712 proteins using the XGBoost classifier. The number of samples identified 1188 is noted in each box.

$1189 \mathrm{~K}$ Classification error matrix of the external validation set (2016, CPTAC, BC 1190 cohort, $n=77$ ) for the 12 proteins using the XGBoost classifier. The number 1191 of samples identified is noted in each box.

1192 L Proteins with the highest predictive values in classifying IBC_Pure and 1193 IBC_LN samples by XGBoost.

1195 Figure 5. Potential sEV survival biomarkers for the distant metastases of 1196 BC.

1197 A The bubble plot indicates the overrepresented pathways in D-MET 1198 compared to DCIS. See Table S5.

1199 B Differentially expressed proteins between distant metastases and DCIS 1200 samples with $>2$-fold difference and two-way Student's $t$ test $p<0.05$.

1201 C Potential markers of distant metastasis were significantly associated with 1202 clinical outcomes in BC (2018, Tang et al., BC cohort, $\mathrm{n}=118)$ ( $p$ value from 1203 log rank test). 
1204 D DAVID (KEGG gene sets) analyses of the proteomic data of $21 \mathrm{BC}$ patients

1205 with distant metastases revealed pathways that were significantly altered in 1206 lung metastases (M-Lung, $n=3$ ), liver metastases (M-Liver, $n=4$ ), and 1207 bone metastases (M-Bone, $\mathrm{n}=7$ ) (Fisher's exact test, $p<0.05$ ).

1208 E GMDS was specifically highly expressed in M-Liver. ns, no significance; ${ }^{*} p<$ $1209 \quad 0.05$ by one-way Student's $t$ test.

1210 F P4HB was specifically highly expressed in M-Bone. ns, no significance; ${ }^{* *} p<$ $1211 \quad 0.01$ by one-way Student's $t$ test.

1212 G C6, TUBB, SERPING1 and VCL were specifically highly expressed in 1213 M-Lung. ns, no significance; ${ }^{*} p<0.05,{ }^{* *} p<0.01$ by one-way Student's $t$ 1214 test.

$1215 \mathrm{H}$ High expression of VCL was associated with poor prognosis in BC (2018, 1216 Tang, et al. BC cohort, $\mathrm{n}=126)$.

1218 Figure 6. Potential BC-derived sEV molecules govern organ-specific 1219 metastasis.

1220 A Distinctive tumour microenvironment of M-Lung, M-Liver, and M-Bone 1221 samples. ${ }^{*} p<0.05,{ }^{* *} p<0.01,{ }^{* *} p<0.001$ by ANOVA.

1222 B Boxplot showing the relative abundance of chondrocytes in the distant 1223 metastases of BC. P value from one-way Student's $t$ test.

1224 C Boxplot showing the relative abundance of myocytes in the distant 1225 metastases of BC. $P$ value from one-way Student's $t$ test.

1226 D Boxplot showing the relative abundance of fibroblasts in the distant 1227 metastases of BC. $P$ value from one-way Student's $t$ test.

1228 E sEV ITGA1, S100A8 and S100A11 molecular levels in M-Bone. P value from 1229 one-way Student's $t$ test.

1230 F Protein expression of ITGA1 in DCIS, M-Liver, M-Lung, and M-Bone tissues 1231 detected by using immunohistochemistry. 
1232 G Protein expression of S100A8 in DCIS, M-Liver, M-Lung, and M-Bone

1233 tissues detected by using immunohistochemistry.

$1234 \mathrm{H}$ Model of sEV-mediated organotropic tumour dissemination. BC-derived

1235 sEVs are taken up by organ-specific resident cells in metastatic organs

1236 based on integrin expression.

1238 Appendix Figure 1. Proteomic characterization of BC-derived sEVs, 1239 related to Figure 1

1240 A NanoSight profiles showing the size distribution of serum-derived sEVs 1241 isolated from BC, BD, and HD. Red denotes BC-derived sEVs, yellow 1242 denotes BD-derived sEVs, and blue denotes HD-derived sEVs.

1243 B Identification of 24 sEV protein markers in our proteomic data.

1244 C Distribution of log10-transformed iBAQ abundance of identified proteins in 1245167 proteome samples that passed quality control. Red denotes BC 1246 samples $(n=126)$, yellow denotes BD samples $(n=17)$, and blue denotes 1247 HD samples $(\mathrm{n}=24)$. In the box plots, the middle bar represents the median, 1248 and the box represents the interquartile range; bars extend to $1.5 \times$ the 1249 interquartile range.

1253 A PCA of 1,734 proteins in 167 samples. Red, BC $(n=126)$; yellow, BD $(n=$ 1254 17); blue, HD ( $\mathrm{n}=24)$.

1255 B Schematic diagram of the structural distribution of damage-associated 1256 molecular patterns (DAMPs) in sEVs (left). Venn diagram showing the 1257 number of DAMPs detected in BC, BD, and HD samples (right). See Table 1258 S2. 
1259 C sEV DAMP molecules enriched in BC were significantly associated with

1260 clinical outcomes in BC (2018, Tang et al., BC cohort, $n=118$ ) ( $p$ value from $1261 \quad \log$ rank test).

1262 D The dataset was split randomly into training (70\%) and test sets (30\%) at the 1263 patient level. A machine learning algorithm, XGBoost, was used for model 1264 development, training, and validation. Receiver operating characteristic (ROC) analysis was used to evaluate the performance of the classifier on the test dataset.

Appendix Figure 3. Proteomic landscapes of four clinical subtypes of BC-derived sEVs, related to Figure 3

A PCA of 1,308 proteins in 114 samples. Orange, luminal A $(n=20)$; green, luminal B $(n=50)$; purple, Her2-enriched $(n=21)$; and blue, TNBC $(n=23)$.

B Proteins with the highest predictive values in classifying luminal A, luminal B, Her2-enriched, and TNBC samples by XGBoost. lymph node metastases, related to Figure 4

1277 A PCA of 1,116 proteins in 105 samples. Blue, invasive breast cancer with 1278 lymph node metastases (IBC_Pure, $n=54$ ); red, invasive breast cancer without lymph node metastases (IBC_LN, $\mathrm{n}=51)$.

1280 B Differentially expressed proteins between IBC_Pure and IBC_LN samples 1281 that were found in $>50 \%$ of the corresponding samples, with $>2$-fold 1282 difference and Student's $t$ test $p<0.05$.

1283 C Comparison of the scores of adipocytes between the IBC_LN group and the 1284 IBC_Pure group. The $p$ value was calculated by the Wilcoxon rank sum test. 1285 The line and box represent median and upper and lower quartiles, respectively. 
1287 D Correlation between adipogenesis and adipocytes. Spearman rho $=0.188, p$ $1288 \quad$ value $=5.507 e-02$

1289 E Comparison of the MPP scores between the IBC_LN group and the 1290 IBC_Pure group. The $p$ value was calculated by the Wilcoxon rank sum test. 1291 The line and box represent the median and upper and lower quartiles, 1292 respectively.

1293 F Correlation between MPPs and the coagulation pathway. Spearman rho = $1294 \quad 0.295, p$ value $=2.216 e-03$

1295 G Correlation between platelets and the coagulation pathway. Spearman rho = $1296 \quad 0.209, p$ value $=3.225 e-02$.

$1297 \mathrm{H}$ Comparison of the platelet scores between the IBC_LN group and the 1298 IBC_Pure group. The $p$ value was calculated by the Wilcoxon rank sum test. 1299 The line and box represent the median and upper and lower quartiles, 1300 respectively.

1301 I Molecules that are highly associated with platelets.

$1302 \mathrm{~J}$ Representative fluorescence microscopy images of MEPs labelled with 1303 CD71 (green), CD38 (red), and CD45RA (yellow). Images revealed the 1304 presence of MEPs in lymph node metastases of $\mathrm{BC}$, which were rare in 1305 normal lymph nodes and primary breast cancer.

1306 K Platelet counts in the blood of IBC patients with lymph node metastasis $(\mathrm{n}=$ 1307 43) and IBC patients without lymph node metastasis ( $n=45)$.

$1308 \mathrm{~L}$ The dataset was randomly split into training (70\%) and test sets (30\%) at the 1309 patient level. A machine learning algorithm, XGBoost, was used for model 1310 development, training, and validation. Receiver operating characteristic 1311 (ROC) analysis was used to evaluate the performance of the classification 1312 on the test dataset. 
Appendix Figure 5. Potential sEV survival biomarkers for the distant metastases of $\mathrm{BC}$, related to Figure 5

A Potential markers of distant metastasis were significantly associated with clinical outcomes in BC (2018, Tang et al., BC cohort, $n=118$ and 2014, Liu et al., $B C$ cohort, $n=126$ ) ( $p$ value from log rank test).

Appendix Figure 6. Potential molecules present on IBC-derived sEVs target them to specific organs, related to Figure 6

A IHC score of ITGA1 in DCIS ( $n=4)$, M-Liver $(n=4)$, M-Lung $(n=4)$, and M-Bone $(\mathrm{n}=8)$. $P$ value from two-way Student's $t$ test.

B IHC score of S100A8 in DCIS ( $n=3)$, M-Liver $(n=3)$, M-Lung $(n=4)$, and M-Bone $(\mathrm{n}=8)$. $P$ value from two-way Student's $t$ test. one-way Student's $t$ test.

D sEV ITGB3, S100A2B and S100A7A molecular levels in M-Lung. $P$ value from one-way Student's $t$ test.

1330 E The protein expression of S100A13 in DCIS $(n=3)$, M-Liver $(n=3)$, M-Lung

1331 ( $\mathrm{n}=4)$, and M-Bone $(\mathrm{n}=8)$ tissues was detected by using 1332 immunohistochemistry (left); IHC score of S100A13 in M-Liver. $P$ value from 1333 two-way Student's $t$ test (right).

1334 F The protein expression of S100A7A in DCIS $(n=3)$, M-Liver $(n=3)$, M-Lung

$1335(\mathrm{n}=4)$, and M-Bone $(\mathrm{n}=8)$ tissues was detected by using 1336 immunohistochemistry (left); IHC score of S100A7A in M-Lung. $P$ value from 1337 two-way Student's $t$ test (right). 
Figure 1

A

Breast Cancer $(\mathrm{BC})$

intiving

$\|$

Benign Disease (BD)

17

Healthy Donor (HD) în
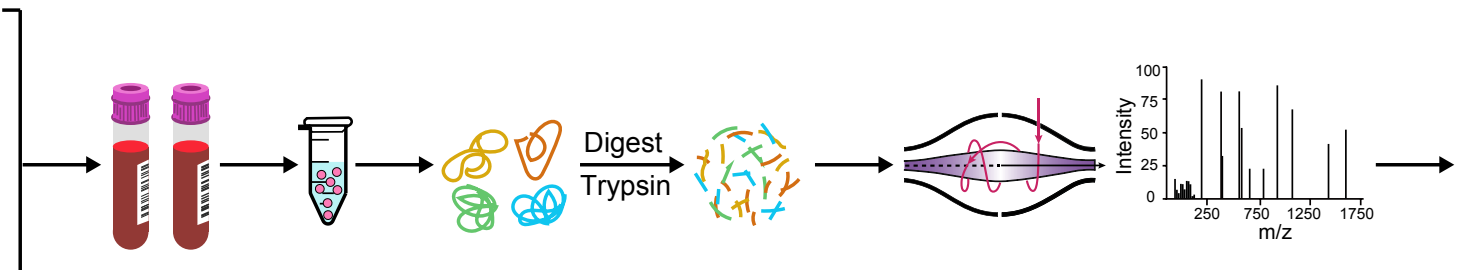

LC-MS/MS \& DIA

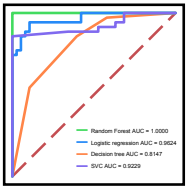

Data analysis \& interpretation

B

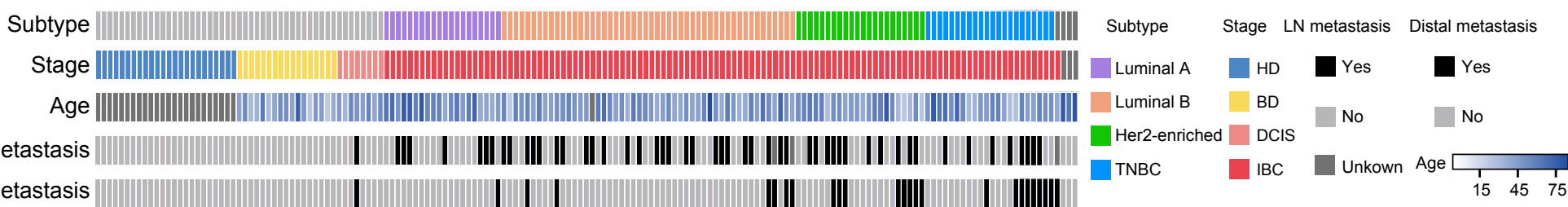

C

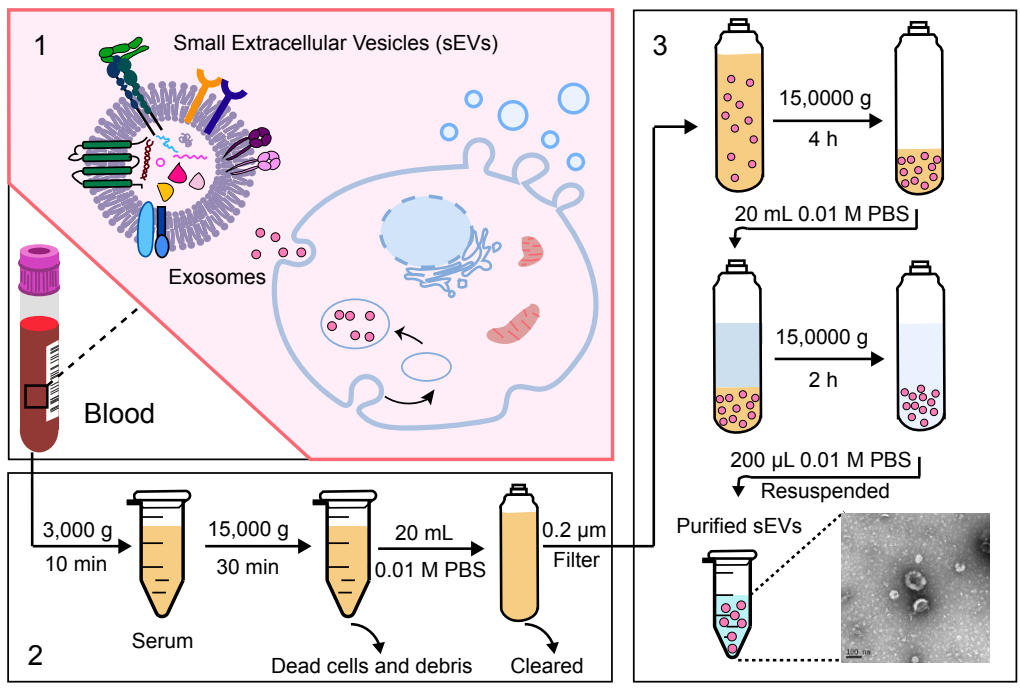

$\mathrm{D}$

Healthy Donor (HD) Benign Disease (BD) Breast Cancer (BC)
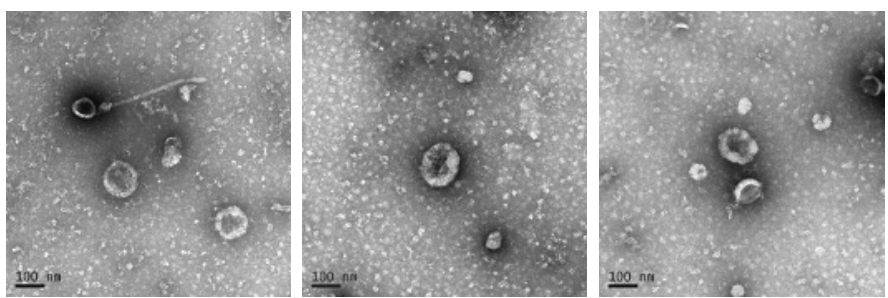

E (PDCD6IP) ALIX

CD63

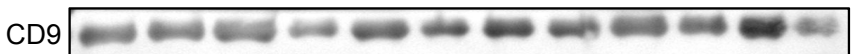

TSG101

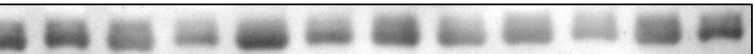

$\mathrm{F}$

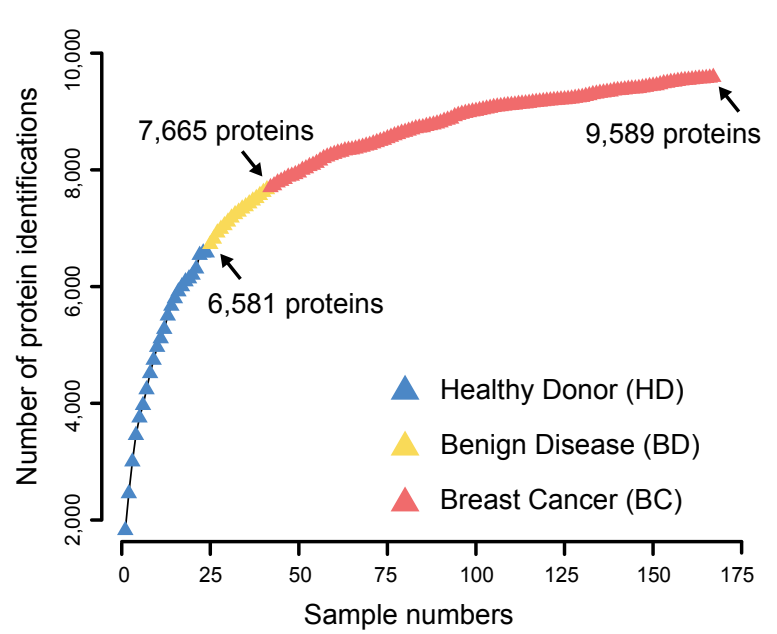

G

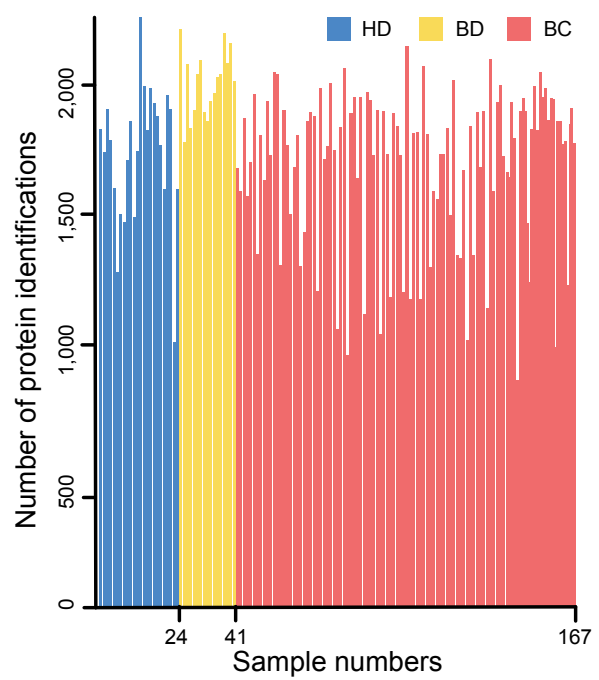

$\mathrm{H}$

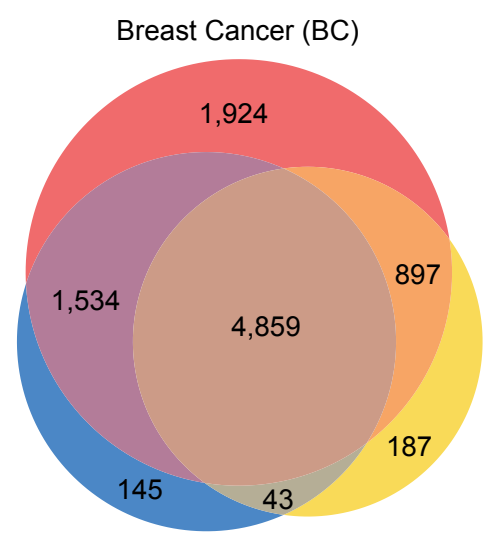

Healthy Donor (HD) Benign Disease (BD) 


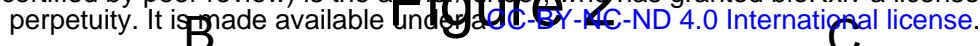

MMP2 (2018, Tang, et al.)

\section{Transport \& Phagocytosis \\ COPI-mediated anterograde transport \\ Vesicle-mediated transport \\ Regulation of actin dynamics for phagocytic cup formation}

\section{Adhesion \& Hormone \\ Cell-cell adhesion \\ Cholesterol metabolic process \\ - Response to estrogen}

Immune \& Metabolism \& Metastasis
$\bigcirc$ Interferon y-mediated signaling pathway
Regulation of immune response
Antigen processing and presentation
$\circ$ Glycolytic process
Angiogenesis

$\widehat{0}$
$\frac{9}{3}$
$\frac{0}{2}$
0
0
0
0
0

D
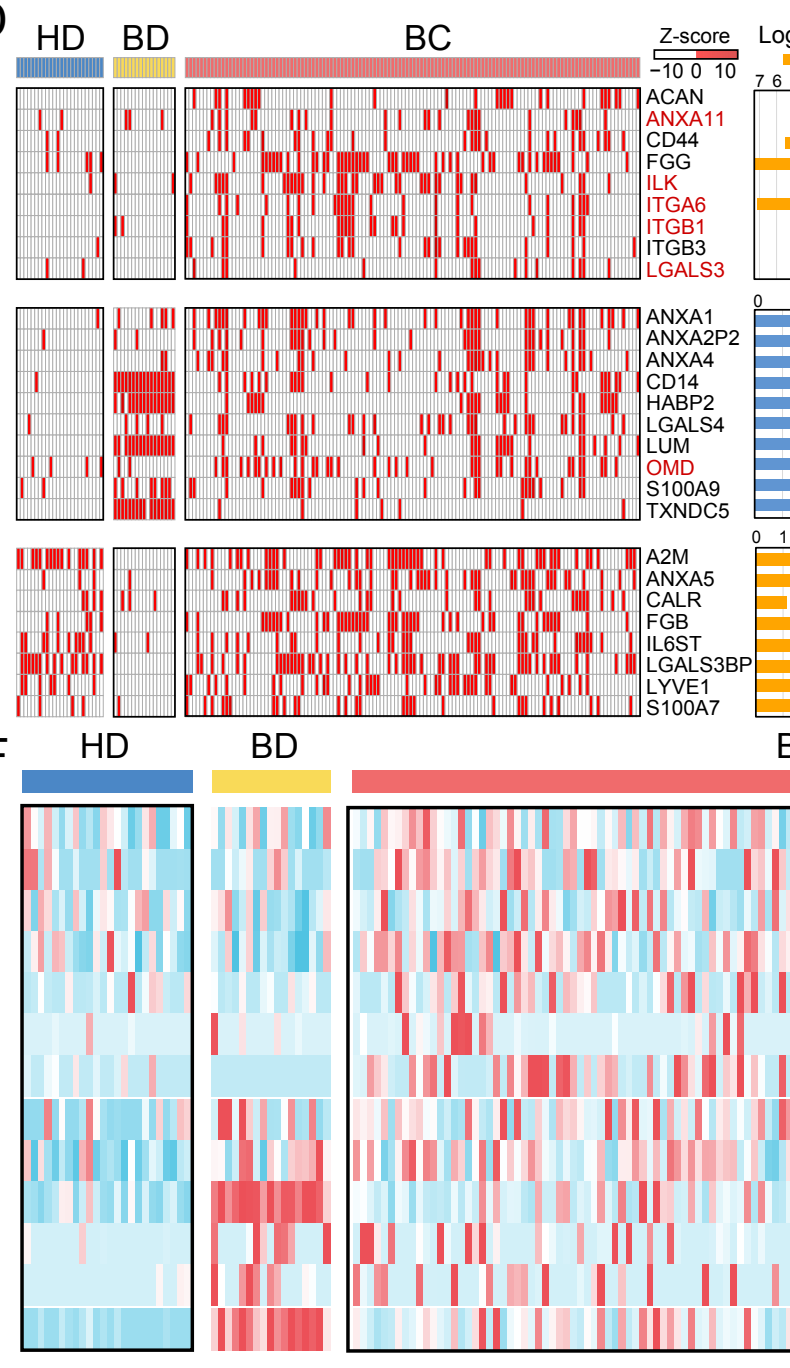

$\mathrm{BC}$

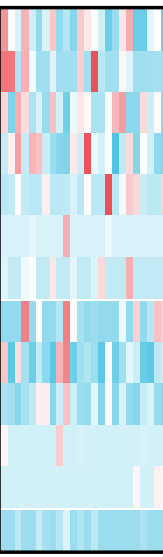

HD BD BC $\quad$ Z-score

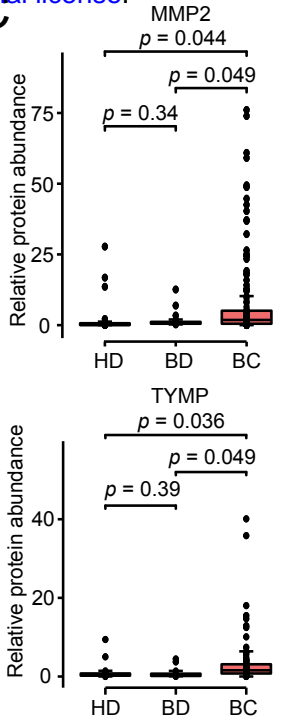

I. ARF5 IGHV3-53
IGHV4-39 SSC5D COPE | PPL24 FNBP1L 11 PON1 APOC2 ANGPTL3
IGFBP2 1 LIPC CYP1B1
TYMP HLA-C MMP2
PVR

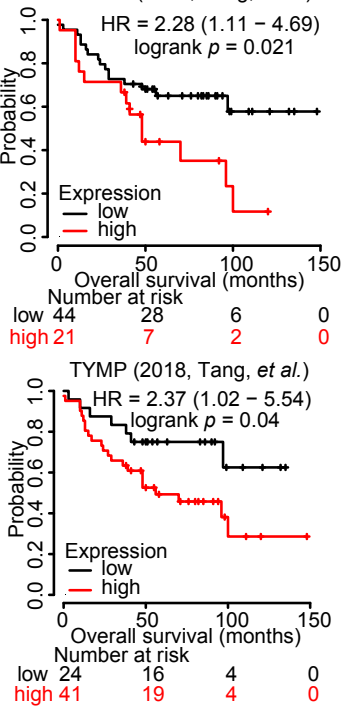

$E$

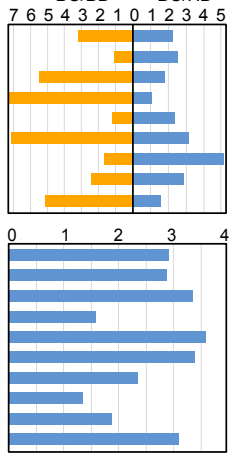

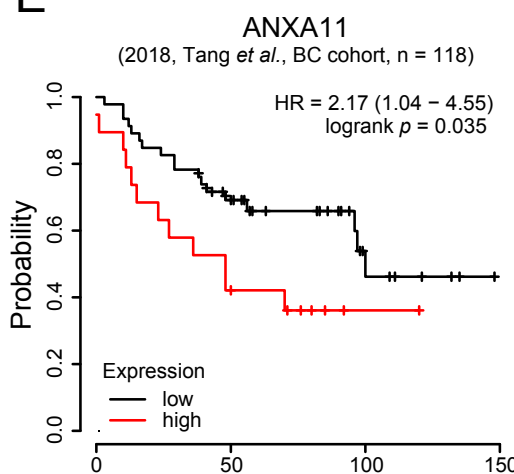

Overall survival (months) Number at risk low $46 \quad 27$ high 19

\section{Z-score
$-1-0.500 .51$}

The function of BC-derived sEVs
(4)

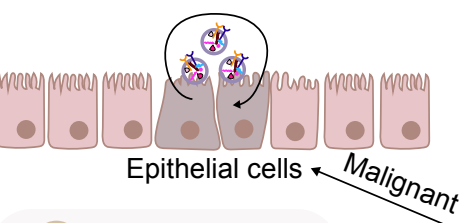

$\mathrm{H}$

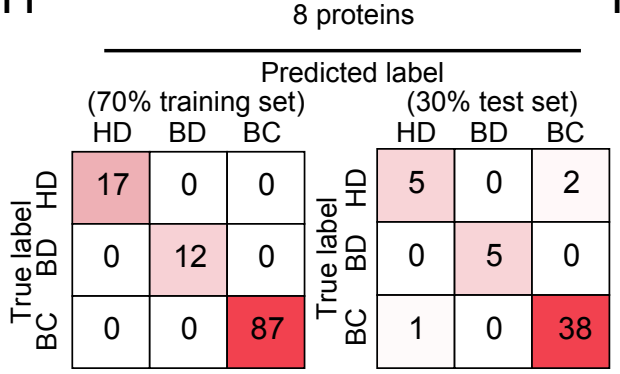

The diagnosis of $\mathrm{BC}$ :

Sensitivity: $100 \%$

Specificity: $100 \%$

Positive Predictive

Value: $100 \%$

Negative Predictive

Value: $100 \%$
Sensitivity: $97 \%$ Specificity: $83 \%$ Positive Predictive Value: $95 \%$

Negative Predictive Value: $91 \%$
ILK

(2018, Tang et al., BC cohort, $\mathrm{n}=118$ )

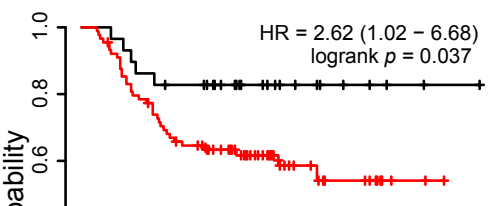

Expression

$\circ$ ○

Overall survival (months) Number at risk

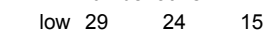

$\begin{array}{cccccc}\text { high } 89 & 63 & 38 & 13 & 2 & 1 \\ \end{array}$

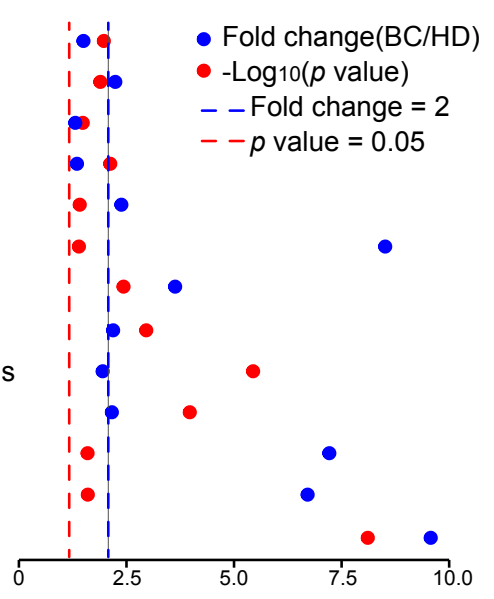

I

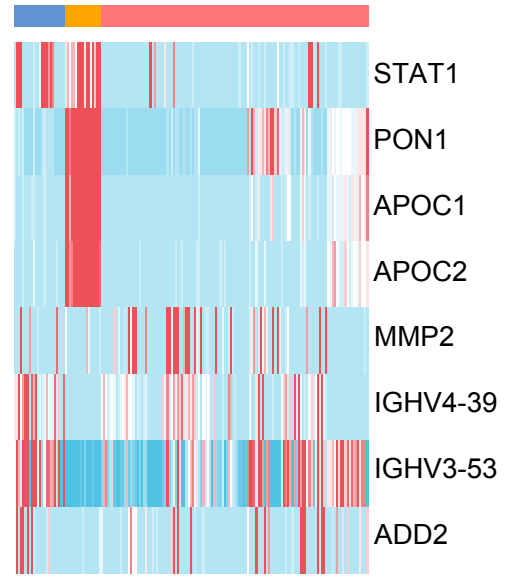


bioRxiv preprint doi: https://doi.org/10.1101/2021.11.26.470104; this version posted November 27, 2021. The copyright holder for this preprint (which was not certified by peer review) is the author/funder, who has granted bioRxiv a license to display the preprint in perpetuity. It is made available under aCC-BY-NC-ND 4.0 International license.

\section{Figure 3}

A

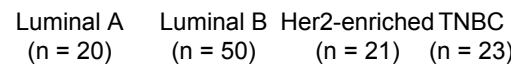
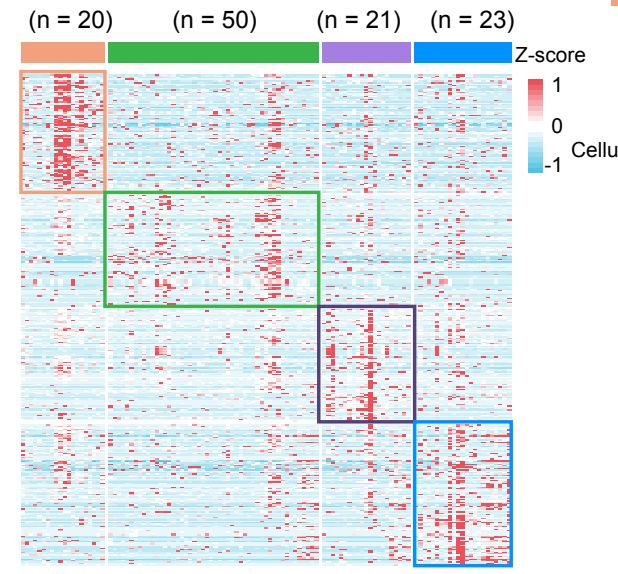

- Luminal A $\quad$ Luminal B $\quad$ Her2-enriched $\quad$ TNBC

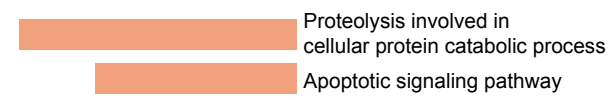

-1 Cellular response to insulin stimulus

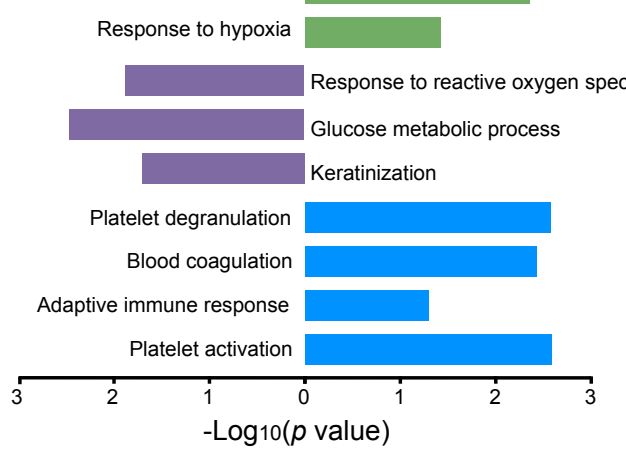

C

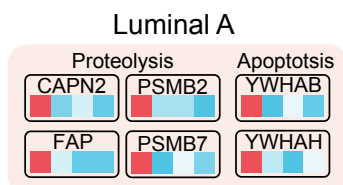

Luminal B

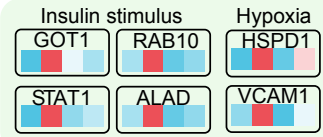

Her2-enriched

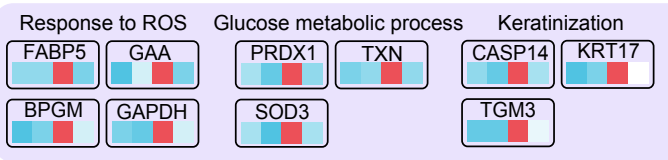

TNBC

Blood coagulation Platelet degranulation Adaptive immune response

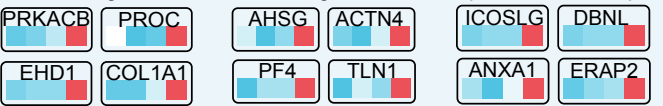

COL1A2 F11 PPBP

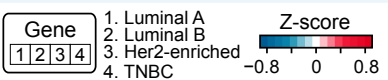

$\mathrm{D}$

61 Proteins

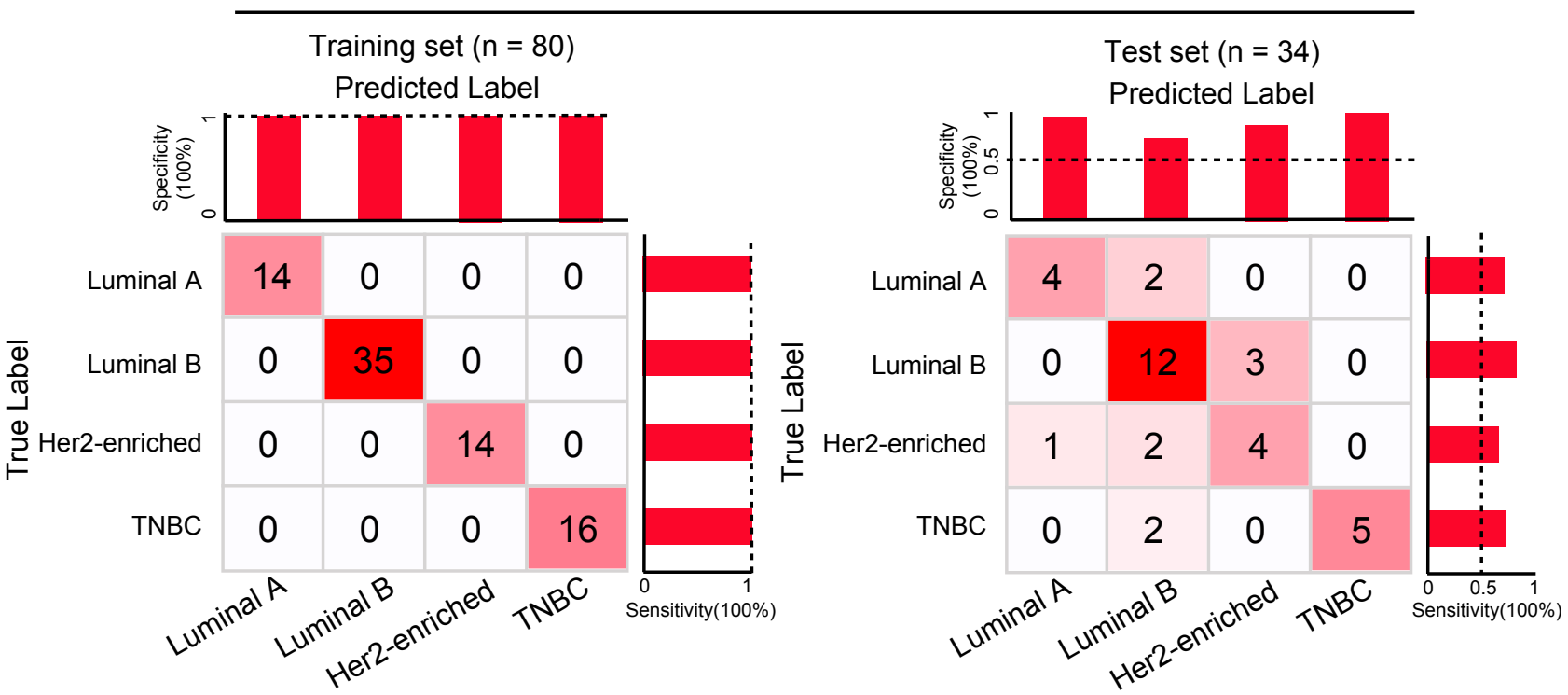

$E$
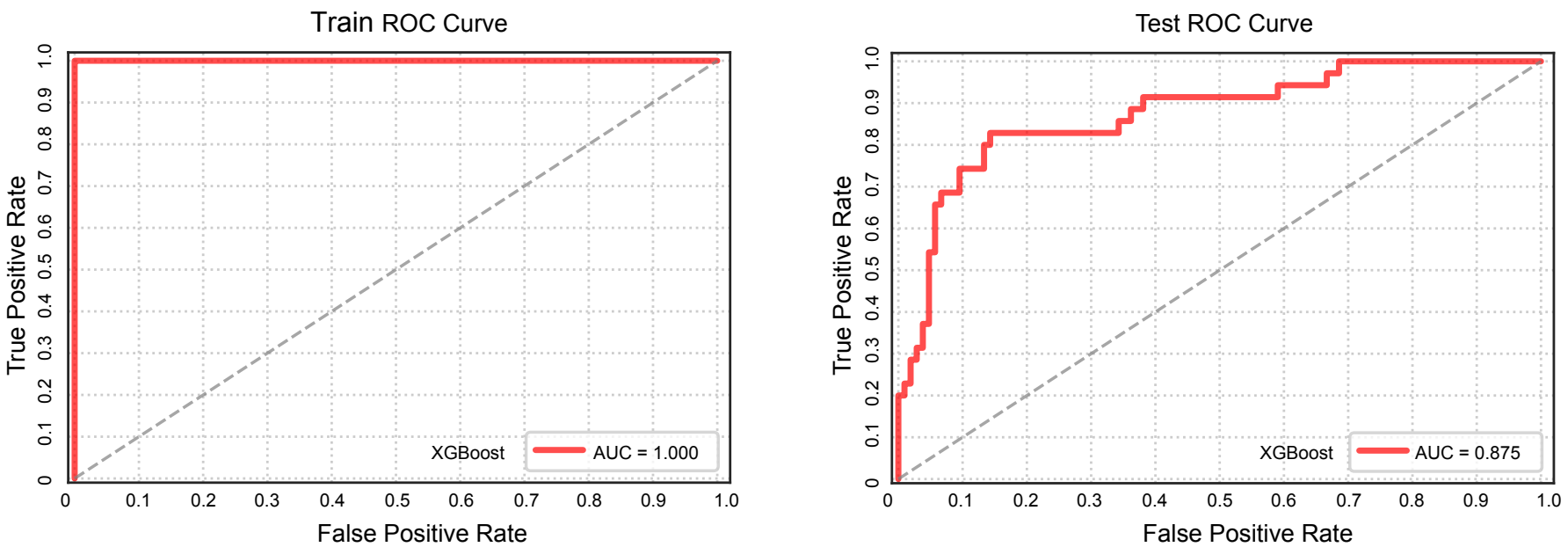
bioRxiv preprint doi: https://doi.org/10.1101/2021.11.26.470104; this version posted November 27, 2021. The copyright holder for this preprint (which was not certified by peer review) is the author/funder, who has granted bioRxiv a license to display the preprint in

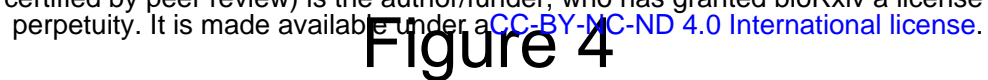

A

HALLMARK_Adipogenesis

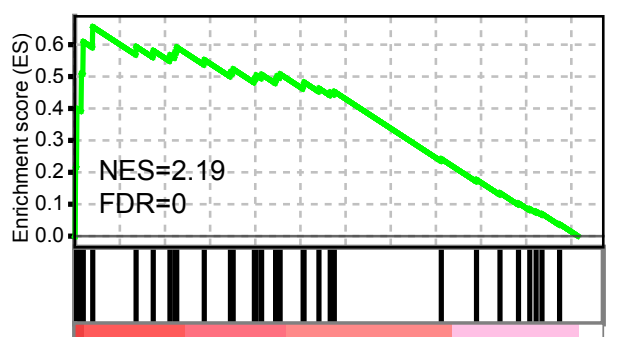

IBC_LN

B

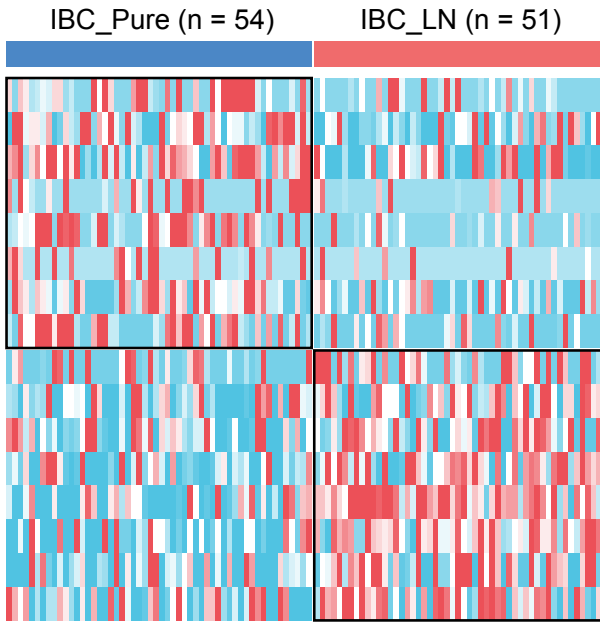

Z-score

B-cells

Basophils

CD4+ T-cells

CD4+ naive T-cells

DC

Mesangial cells

aDC

iDC

Adipocytes

CD8+ T-cells

CD8+ naive $\mathrm{T}$-cells MPPs

Macrophages

Megakaryocytes

Platelets

Sebocytes
C

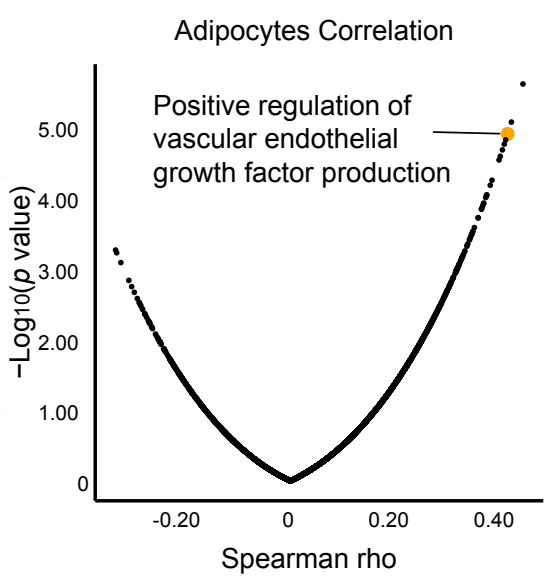

G

F
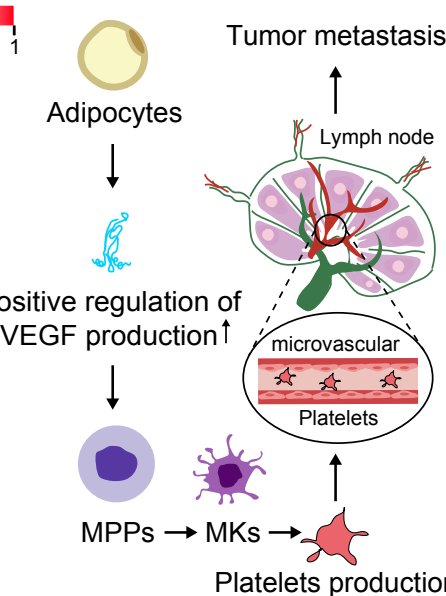

Platelets production

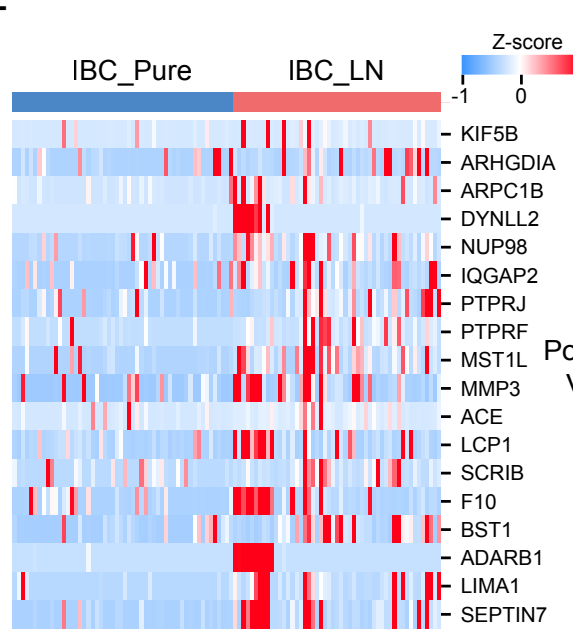

Positive regulation of VEGF production Correlation
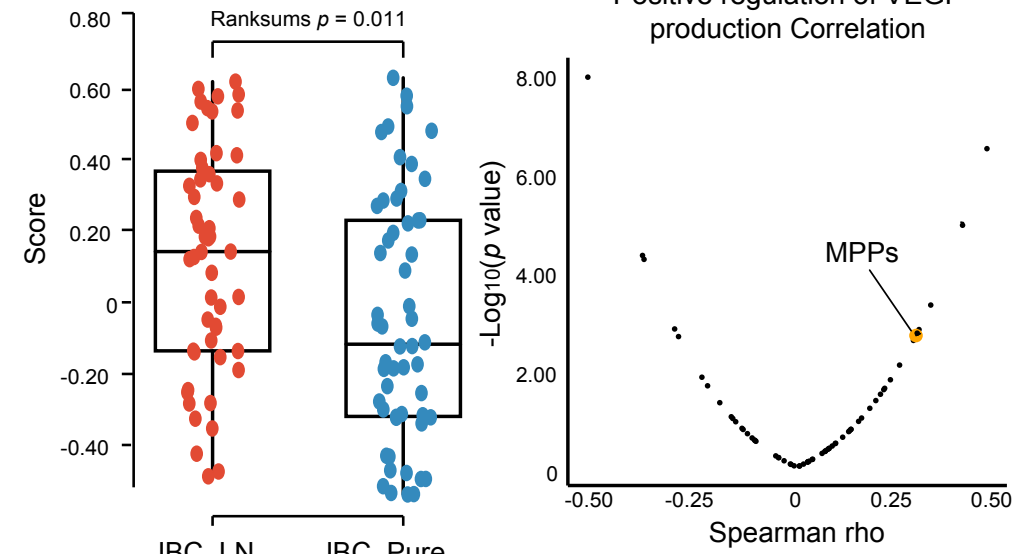

$\mathrm{H}$


$$
J
$$

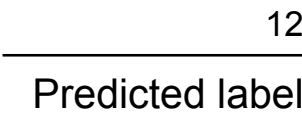

(70\% training set)

IBC_Pure IBC_LN

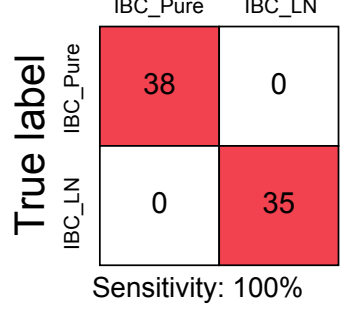

Specificity: $100 \%$

Positive Predictive Value: $100 \%$

Negative Predictive Value: 100\%
Predicted label

(30\% test set)

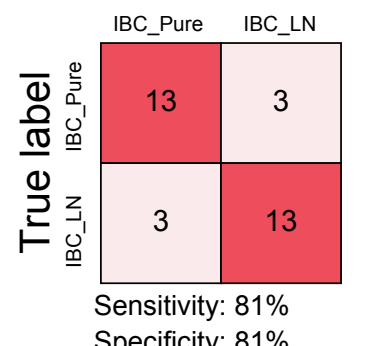

Specificity: $81 \%$

Positive Predictive Value: $81 \%$ Negative Predictive Value: $81 \%$
K

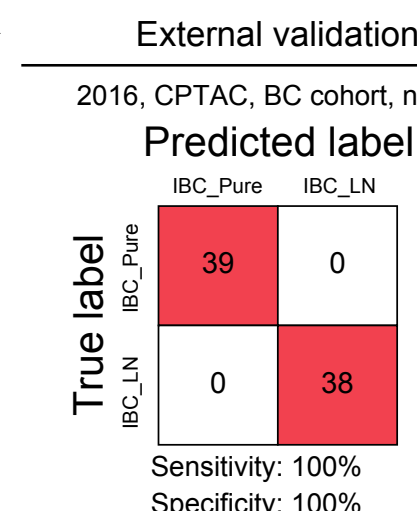

Positive Predictive Value: 100\% Negative Predictive Value: $100 \%$
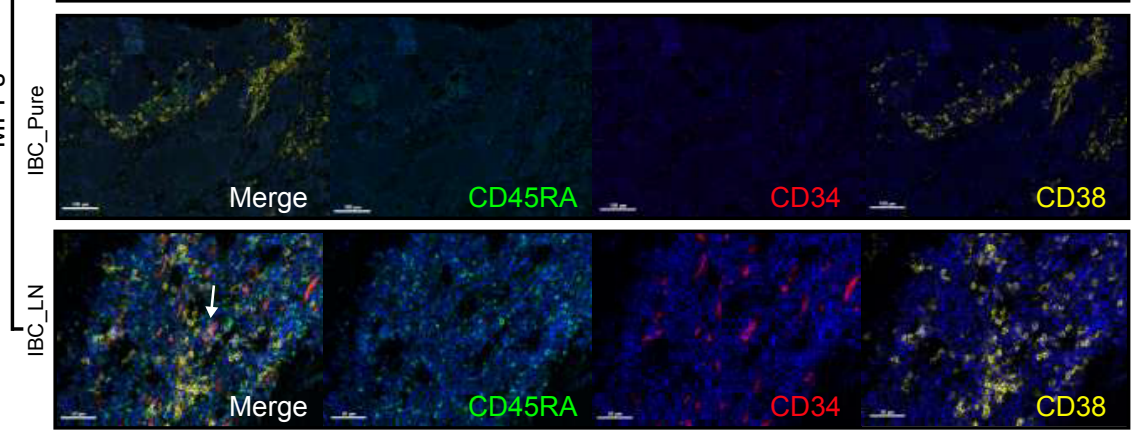

$\mathrm{L}$

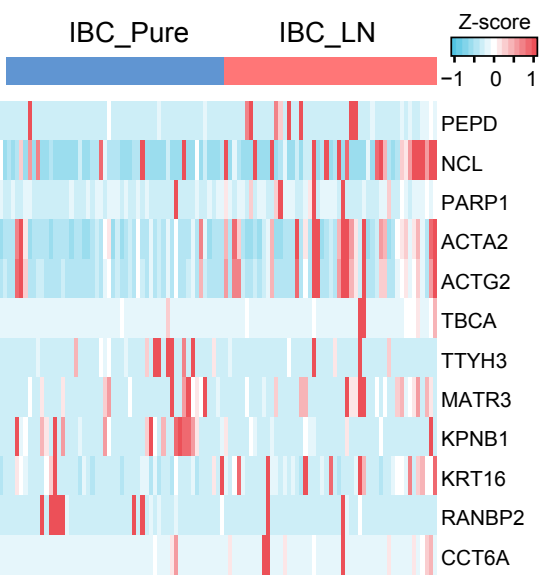


A

DCIS M-Lung M-Liver M-Bone

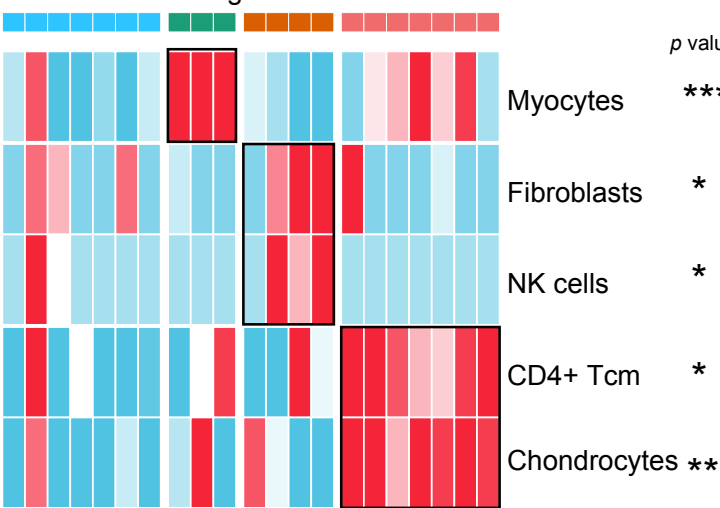

$\mathrm{D}$

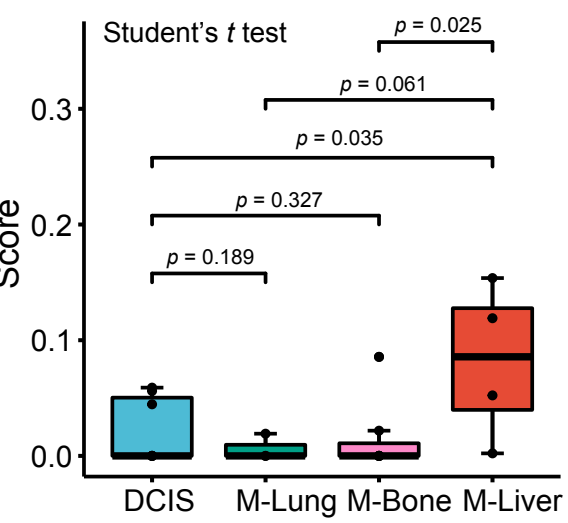

$\mathrm{F}$

¿
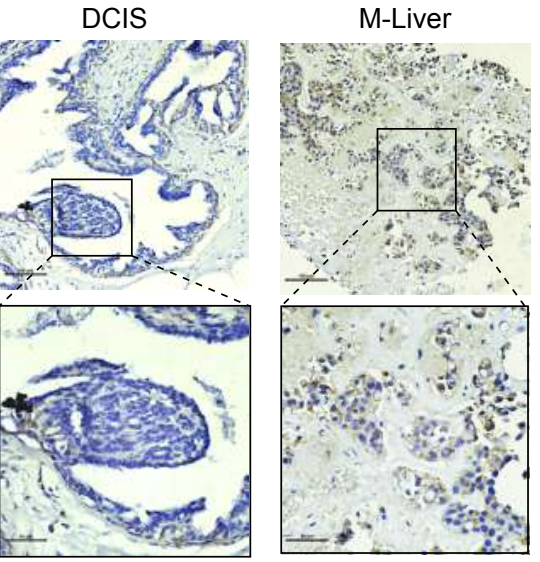

G

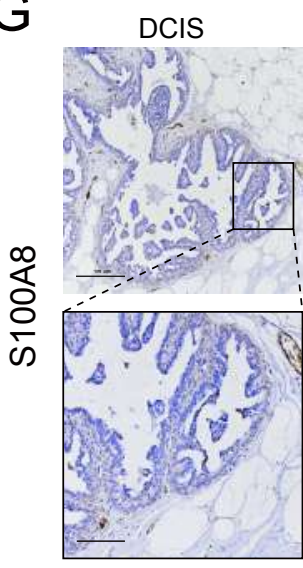

B

Chondrocytes

$E$
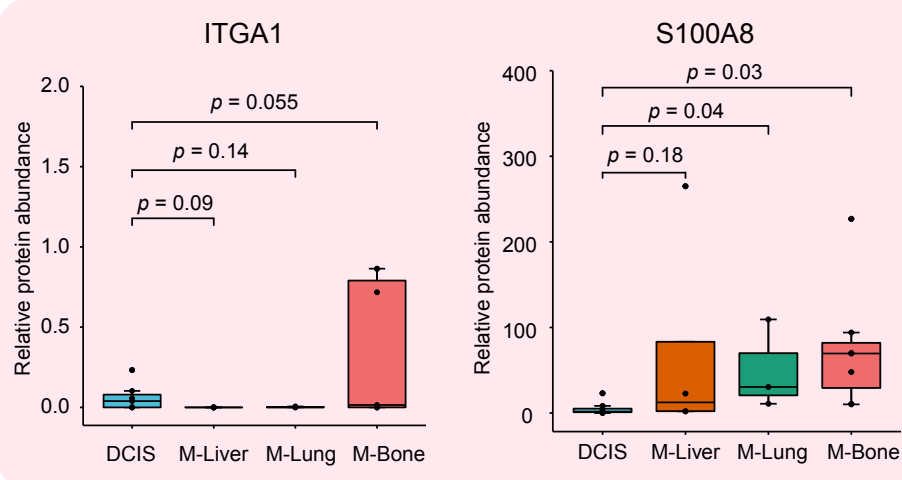

C

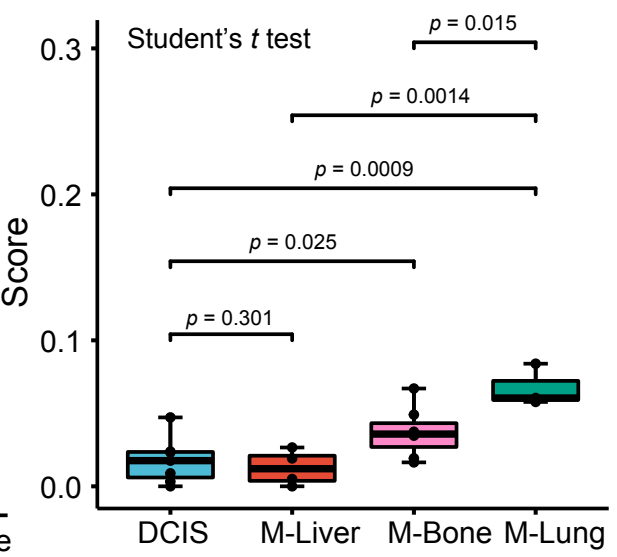

Myocytes

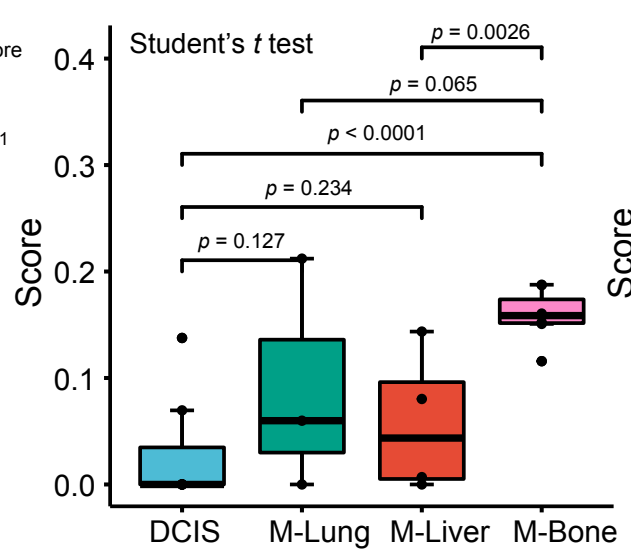

